\title{
NEXP-completeness and Universal Hardness Results for Justification Logic
}

\author{
Antonis Achilleos \\ The Graduate Center of CUNY \\ 365 Fifth Avenue \\ New York, NY 10016 USA \\ aachilleos@gc. cuny.edu
}

\begin{abstract}
We provide a lower complexity bound for the satisfiability problem of a multi-agent justification logic, establishing that the general NEXP upper bound from our previous work is tight. We then use a simple modification of the corresponding reduction to prove that satisfiability for all multi-agent justification logics from there is $\Sigma_{2}^{p}$-hard - given certain reasonable conditions. Our methods improve on these required conditions for the same lower bound for the single-agent justification logics, proven by Buss and Kuznets in 2009, thus answering one of their open questions.
\end{abstract}

\section{Introduction}

Justification Logic is the logic of justifications. Where in Modal Epistemic Logic we use formulas of the form $\square \phi$ to denote that $\phi$ is known (or believed, etc), in Justification Logic, we use $t: \phi$ to denote that $\phi$ is known for reason $t$ (i.e. $t$ is a justification for $\phi)$. Artemov introduced LP, the first justification logic, in 1995 [5], originally as a link between Intuitionistic Logic and Peano Arithmetic. Since then the field has expanded significantly, both in the variety of logical systems and in the fields it interacts with and is applied to (see $[6,7]$ for an overview).

In [21] Yavorskaya introduced two-agent LP with agents whose justifications may interact. We studied the complexity of a generalization in [3] and [4], discovering that unlike the case with single-agent Justification Logic as studied in $[12,13,15,8,1]$, the complexity of satisfiability jumps to PSPACE- and EXPcompleteness when two or three agents are involved respectively, given appropriate interactions. In fact, the upper bound we proved was that all logics in this family have their satisfiability problem in NEXP - under reasonable assumptions.

The NEXP upper complexity bound was not met with the introduction of a NEXP-hard logic in [4]. The main contribution of this paper is that we present a NEXP-hard justification logic from the family that was introduced in [4], thus establishing that the general upper bound is tight.

In general, the complexity of the satisfiability problem for a justification logic tends to be lower than the complexity of its corresponding modal $\operatorname{logic}^{1}$ (given

\footnotetext{
${ }^{1}$ That is, the modal logic that is the result of substituting all justification terms in the axioms with boxes and adding the Necessitation rule.
} 
the usual complexity-theoretic assumptions). For example, while satisfiability for $\mathrm{K}, \mathrm{D}, \mathrm{K} 4, \mathrm{D} 4, \mathrm{~T}$, and S4 is PSPACE-complete, the complexity of the corresponding justification logics (J, JD, J4, JD4, JT, and LP respectively) is in the second level of the polynomial hierarchy (in $\Sigma_{2}^{p}$, specifically). In the multi-agent setting we have already examined, this is still the case: many justification logics that so far demonstrate a complexity jump (to PSPACE- or EXP-completeness) have corresponding modal logics with an EXP-complete satisfiability problem (c.f. $[20,9,1,2])$. It is notable that, assuming EXP $\neq$ NEXP, this is the first time we have a justification logic with a higher complexity than its corresponding modal logic, and, in fact, the reduction we use makes heavy use of the effects of the way a justification term is constructed.

In a justification logic, the logic's axioms are justified by constants, a kind of minimal (not analyzable) justification. A constant specification is part of the description of a justification logic and specifies exactly which constants justify which axioms. There are certain standard assumptions we often need to make when studying the complexity of a justification logic. One is that the logic has an axiomatically appropriate constant specification, which means that all axioms of the logic are justified by at least one justification constant. Another is that the logic has a schematic constant specification, which means that each constant justifies a certain number of axiom schemes (perhaps none) and nothing else. Finally, the third assumption is that the constant specification is schematically injective, that is, it is schematic and each constant justifies at most one scheme.

It is known that for (single-agent) justification logics J, JT, J4, and LP, the satisfiability problem is in $\Sigma_{2}^{p}$ for a schematic constant specification ([12]) and for JD, JD4, the satisfiability problem is in $\Sigma_{2}^{p}$ for an axiomatically appropriate and schematic constant specification $([13,1])$. As for the lower bounds, Milnikel has proven ([17]) that J4-satisfiability is $\Sigma_{2}^{p}$-hard for an axiomatically appropriate and schematic constant specification and that LP-satisfiability is $\Sigma_{2}^{p}$-hard for an axiomatically appropriate, (schematic,) and schematically injective constant specification. Following that, Buss and Kuznets gave a general lower bound in [8], proving that for all the above logics, satisfiability is $\Sigma_{2}^{p}$-hard for an axiomatically appropriate, (schematic,) and schematically injective constant specification. This raised the question of whether the condition that the constant specification is schematically injective is a necessary one, which is answered in this paper. ${ }^{2}$

We present a general lower bound, which applies to all logics from [4]. This includes all the single-agent logics whose complexity was studied in $[12,13,8,1]$. In fact, Buss and Kuznets gave the same general lower bound for all the singleagent cases in [8] and it is reasonable to expect that we could simply apply their techniques and achieve the same result in this general multi-agent setting. Our method, however, presents the following two advantages: it is a relatively simple reduction, a direct simplification of the more involved NEXP-hardness reduction and very similar to Milnikel's method from [17]; it is also an improvement of their result, even if it does not improve the bound itself in that for our results the requirements are that the constant specification is axiomatically appropriate

\footnotetext{
2 The answer is 'no'.
} 
and schematic - and not that it is schematically injective as well. In particular this means that we provide for the first time a tight lower bound for the full LP (LP where all axioms are justified by all constants). The disadvantage of our method is that, unlike the one of Buss and Kuznets, it cannot be adjusted to work on the reflected fragments of justification logic, the fragment which includes only the formulas of the form $t: \phi$.

\section{$2 \quad$ Background}

We present the family of multiagent justification logics from [4], its semantics and $*$-calculus, and notation we will be using. The definitions and propositions in this section can be found in $[3,4]$.

\subsection{Syntax and Axioms}

The justification terms of the language $L_{n}$ include constants $c_{1}, c_{2}, c_{3}, \ldots$ and variables $x_{1}, x_{2}, x_{3}, \ldots$ and $t::=x|c|[t+t]|[t \cdot t]| ! t$. The set of terms is called $T m$. The $n$ agents are represented by the positive integers $i \in N=\{1, \ldots, n\}$. The propositional variables will usually (but not always, as will be evident in the following section) be $p_{1}, p_{2}, \ldots$ Formulas of the language $L_{n}$ are defined: $\phi::=\perp|p| \neg \phi|\phi \rightarrow \phi| \phi \wedge \phi|\phi \vee \phi| t:_{i} \phi$, but depending on convenience we may treat some connectives as constructed from others. We are particularly interested in $r L_{n}=\left\{t:_{i} \phi \in L_{n}\right\}$. Intuitively, · applies a justification for a statement $A \rightarrow B$ to a justification for $A$ and gives a justification for $B$. Using + we can combine two justifications and have a justification for anything that can be justified by any of the two initial terms - much like the concatenation of two proofs. Finally, ! is a unary operator called the proof checker. Given a justification $t$ for $\phi$, ! justifies the fact that $t$ is a justification for $\phi$.

If $\subset, \hookrightarrow$ are binary relations on the agent set $\{1, \ldots, n\}$ and for every agent $i, F(i)$ is a (single-agent) justification logic (we assume $F(i) \in\{\mathrm{J}, \mathrm{JD}, \mathrm{JT}\}$ ), then justification $\operatorname{logic} J=(n, \subset, \hookrightarrow, F)_{\mathcal{C S}}$ has the axioms as seen on Table 2.1 and modus ponens. The binary relations $\subset, \hookrightarrow$ determine the interactions among the agents: $\subset$ determines the instances of the Conversion axiom, while $\hookrightarrow$ the instances of the Verification axiom, so if $i \subset i$, then the justifications of agent $j$ are also valid justifications for agent $i$ (i.e. we have axiom $t:_{j} \phi \rightarrow t:_{i} \phi$ ), while if $i \hookrightarrow i$, then the justifications of agent $j$ can be verified by agent $i$ (i.e. we have axiom $\left.t:{ }_{j} \phi \rightarrow ! t:{ }_{i} t:_{j} \phi\right)$. $F$ assigns a single-agent justification logic to each agent. We would assume $F(i)$ is one of J, JD, JT, J4, JD4, and LP, but since Positive introspection is a special case of Verification, we can limit the choices for $F(i)$ to logics without Positive Introspection (i.e. J, JD, and JT). $\mathcal{C S}$ is called a constant specification. It introduces justifications for the axioms and is explained in Table 2.1 together with the axioms. We also define $i \supset j$ iff $j \subset i$ and $i \hookleftarrow j$ iff $j \hookrightarrow i$.

In this paper we will be making the assumption that the constant specifications are axiomatically appropriate: each axiom is justified by at least one constant; and schematic: every constant justifies only a certain number (0 or 
General axioms (for every agent $i$ ):

Propositional Axioms: Finitely many schemes of classical propositional logic;

Application: $s:{ }_{i}(\phi \rightarrow \psi) \rightarrow\left(t:_{i} \phi \rightarrow[s \cdot t]:_{i} \psi\right)$;

Concatenation: $s:_{i} \phi \rightarrow[s+t]:_{i} \phi, s:_{i} \phi \rightarrow[t+s]:_{i} \phi$.

Agent-dependent axioms (depending on $F(i)$ ):

Factivity: for every agent $i$, such that $F(i)=\mathrm{JT}, t:_{i} \phi \rightarrow \phi$;

Consistency: for every agent $i$, such that $F(i)=\mathrm{JD}, t: t_{i} \perp \rightarrow \perp$.

Interaction axioms (depending on the binary relations $\subset$ and $\hookrightarrow$ ):

Conversion: for every $i \supset j, t:_{i} \phi \rightarrow t:_{j} \phi$;

Verification: for every $i \hookleftarrow j, t: i \phi \rightarrow ! t:{ }_{j} t:{ }_{i} \phi$.

A constant specification for $(n, \subset, \hookrightarrow, F)$ is any set of formulas of the form $c:_{i} A$, where $c$ a justification constant, $i$ an agent, and $A$ an axiom of the logic from the ones above. We say that axiom $A$ is justified by a constant $c$ for agent $i$ when $c:_{i} A \in \mathcal{C S}$.

Axiom Necessitation (AN): $t:_{i} \phi$, where either $t:_{i} \phi \in \mathcal{C S}$ or $t=! s$ and $\phi=s:_{j} \psi$ an instance of Axiom Necessitation.

Table 1. The axioms of $(n, \subset, \hookrightarrow, F)_{\mathcal{C S}}$

more) of the logic's axiom schemes (Table 2.1) - as a result, every constant justifies a finite number of axiom schemes, but either 0 or infinite axioms, while if $c$ justifies $A$ for $i$ and $B$ results from $A$ and substitution, then $c$ justifies $B$ for $i$.

We use the following notation and conventions: For justification terms $t_{1}, \ldots, t_{k}$ and formulas $\phi_{1}, \ldots, \phi_{k}$, term $\left[t_{1}+t_{2}+\cdots+t_{k}\right]$ is defined as $\left[\left[t_{1}+t_{2}+\cdots+t_{k-1}\right]+t_{k}\right]$, $\left[t_{1} \cdot t_{2} \cdots t_{k}\right]$ is defined as $\left[\left[t_{1} \cdot t_{2} \cdots t_{k-1}\right] \cdot t_{k}\right]$, and $\left(\phi_{1} \wedge \phi_{2} \wedge \cdots \wedge \phi_{k}\right)$ as $\left(\left(\phi_{1} \wedge \phi_{2} \wedge \cdots \wedge \phi_{k-1}\right) \wedge \phi_{k}\right)$ when $k>2$. We often identify conjunctions of formulas with sets of such formulas, as long as these can be used interchangeably. For set of indexes $A$ and $\Phi=\left\{t_{a}: i_{a} \phi_{a} \mid a \in A\right\}$, we define $\Phi^{\#_{i}}=\left\{\phi_{a} \mid a \in A, i_{a}=i\right\}$ and $* \Phi=\left\{*_{i_{a}}\left(t_{a}, \phi_{a}\right) \mid a \in A\right\}$. Often we identify 0,1 with $\perp, \top$ respectively, as long as it is not a source of confusion.

Lemma 1 (Internalization Property, [4], but originally [5]). For an axiomatically appropriate constant specification $\mathcal{C S}$, if $\vdash \phi$, then for any $i \in N$ there is some term $t$ such that for any $\phi^{\prime}$ substitution instance of $\phi, \vdash t:_{i} \phi^{\prime}$.

Proof (Quick sketch). By induction on the proof of $\phi$ : easy by AN if $\phi$ is an axiom and using the application axiom if $\phi$ is the result of modus ponens.

The Internalization Property demonstrates three important points. One is that a theorem's proof can be internalized as a justification for that theorem. Another point is that Modal Logic's Necessitation rule survives in Justification Logic - in a weakened form as an axiom and in its full form as a property of 
the logic. The third point is the importance of the assumption that the constant specification is axiomatically appropriate as it is necessary for the lemma's proof.

\subsection{Semantics}

We present Fitting (F-) models for $J=(n, \subset, \hookrightarrow, F)_{\mathcal{C S}}$. These are Kripke models with an additional machinery (an admissible evidence function) to accommodate justification terms. They were introduced by Fitting in [10] with variations appearing in $[19,14]$.

Definition 1. An F-model $\mathcal{M}$ for $J$ is a quadruple $\left(W,\left(R_{i}\right)_{i \in N},\left(\mathcal{E}_{i}\right)_{i \in N}, \mathcal{V}\right)$, where $W \neq \emptyset$ is a set, for every $i \in N, R_{i} \subseteq W^{2}$ is a binary relation on $W$, $\mathcal{V}:$ Pvar $\longrightarrow 2^{W}$ and for every $i \in N, \mathcal{E}_{i}:\left(T m \times L_{n}\right) \longrightarrow 2^{W} . W$ is called the universe of $\mathcal{M}$ and its elements are the worlds or states of the model. $\mathcal{V}$ assigns a subset of $W$ to each propositional variable, $p$, and $\mathcal{E}_{i}$ assigns a subset of $W$ to each pair of a justification term and a formula. $\left(\mathcal{E}_{i}\right)_{i \in N}$ is often seen and referred to as $\mathcal{E}: N \times T m \times L_{n} \longrightarrow 2^{W}$ and $\mathcal{E}$ is called an admissible evidence function (aef). Additionally, for any $i \in N$, formulas $\phi, \psi$, and justification terms $t, s, \mathcal{E}$ and $\left(R_{i}\right)_{i \in N}$ must satisfy the following conditions:

Application closure: $\mathcal{E}_{i}(s, \phi \rightarrow \psi) \cap \mathcal{E}_{i}(t, \phi) \subseteq \mathcal{E}_{i}(s \cdot t, \psi)$.

Sum closure: $\mathcal{E}_{i}(t, \phi) \cup \mathcal{E}_{i}(s, \phi) \subseteq \mathcal{E}_{i}(t+s, \phi)$.

$A N$-closure: for any instance of $A N, t:_{i} \phi, \mathcal{E}_{i}(t, \phi)=W$.

Verification Closure: If $i \hookrightarrow j$, then $\mathcal{E}_{j}(t, \phi) \subseteq \mathcal{E}_{i}\left(! t, t:_{i} \phi\right)$

Conversion Closure: If $i \subset j$, then $\mathcal{E}_{j}(t, \phi) \subseteq \mathcal{E}_{i}(t, \phi)$

Distribution: for $j \hookrightarrow i$ and $a, b \in W$, if $a R_{j} b$ and $a \in \mathcal{E}_{i}(t, \phi)$, then $b \in \mathcal{E}_{i}(t, \phi){ }^{3}$

- If $F(i)=\mathrm{JT}$, then $R_{i}$ must be reflexive.

- If $F(i)=\mathrm{JD}$, then $R_{i}$ must be serial $\left(\forall a \in W \exists b \in W a R_{i} b\right)$.

- If $i \hookrightarrow j$, then for any $a, b, c \in W$, if $a R_{i} b R_{j} c$, we also have $a R_{j} c{ }^{4}$

- For any $i \subset j, R_{i} \subseteq R_{j}$.

Truth in the model is defined in the following way, given a state a:

$-\mathcal{M}, a \not \models \perp$ and if $p$ is a propositional variable, then $\mathcal{M}, a \models p$ iff $a \in \mathcal{V}(p)$.

$-\mathcal{M}, a \models \phi \rightarrow \psi$ if and only if $\mathcal{M}, a \models \psi$, or $\mathcal{M}, a \not \models \phi$.

$-\mathcal{M}, a \models t:_{i} \phi$ if and only if $a \in \mathcal{E}_{i}(t, \phi)$ and $\mathcal{M}, b \models \phi$ for all $a R_{i} b$.

A formula $\phi$ is called satisfiable if there are $\mathcal{M}, a \models \phi$; we then say that $\mathcal{M}$ satisfies $\phi$ in $a$. A pair $\left(W,\left(R_{i}\right)_{i \in N}\right)$ as above is a frame for $(n, \subset, \hookrightarrow, F)_{\mathcal{C S}}$. We say that $\mathcal{M}$ has the Strong Evidence Property when $\mathcal{M}, a \models t:_{i} \phi$ iff $a \in \mathcal{E}_{i}(t, \phi)$. $J$ is sound and complete with respect to its F-models $;^{5}$ it is also complete with respect to F-models with the Strong Evidence property. Furthermore, $J$ has a

\footnotetext{
${ }^{3}$ If we have $\mathcal{M}, a \models t:{ }_{i} \phi-$ and thus $a \in \mathcal{E}_{i}(t, \phi)$ - we also want $\mathcal{M}, a \models ! t:{ }_{j} t:_{i} \phi$ to happen and therefore also $\mathcal{M}, b \models t:{ }_{i} \phi-$ so $b \in \mathcal{E}_{i}(t, \phi)$ must be the case as well.

${ }^{4}$ Thus, if $i$ has positive introspection (i.e. $i \hookrightarrow i$ ), then $R_{i}$ is transitive.

${ }^{5}$ That $\mathcal{C S}$ is axiomatically appropriate is a requirement for completeness.
} 
"small" model property, as Proposition 1 demonstrates. Completeness is proven in $[3,4]$ by a canonical model construction with maximally consistent sets of formulas as states; Proposition 1 is then proven by a modification of that canonical model construction that depends on the particular satisfiable formula $\phi$.

Proposition $1([3,4])$. If $\phi$ is J-satisfiable, then $\phi$ is satisfiable by an F-model for $J$ of at most $2^{|\phi|}$ states which has the strong evidence property.

\subsection{The $*$-calculus.}

The $*$-calculus gives an axiomatization of $r J=\left\{\phi \in r L_{n} \mid J \vdash \phi\right\}$, the reflected fragment of $J$. It is an invaluable tool in the study of the complexity of Justification Logic and when we handle aefs and formulas in $r L_{n}$. A $*$-calculus was introduced in [11], but its origins can be found in [18].

If $t$ is a term, $\phi$ is a formula, and $i \in N$, then $*_{i}(t, \phi)$ is a $*$-expression. Given a frame $\mathcal{F}=\left(W,\left(R_{i}\right)_{i \in N}\right)$ for $J$, the $*^{\mathcal{F}}$-calculus for $J$ is the derivation system on $*$-expressions prefixed by states from $W\left(*^{\mathcal{F}}\right.$-expressions from now on) with the axioms and rules that are shown in Table 2.3.

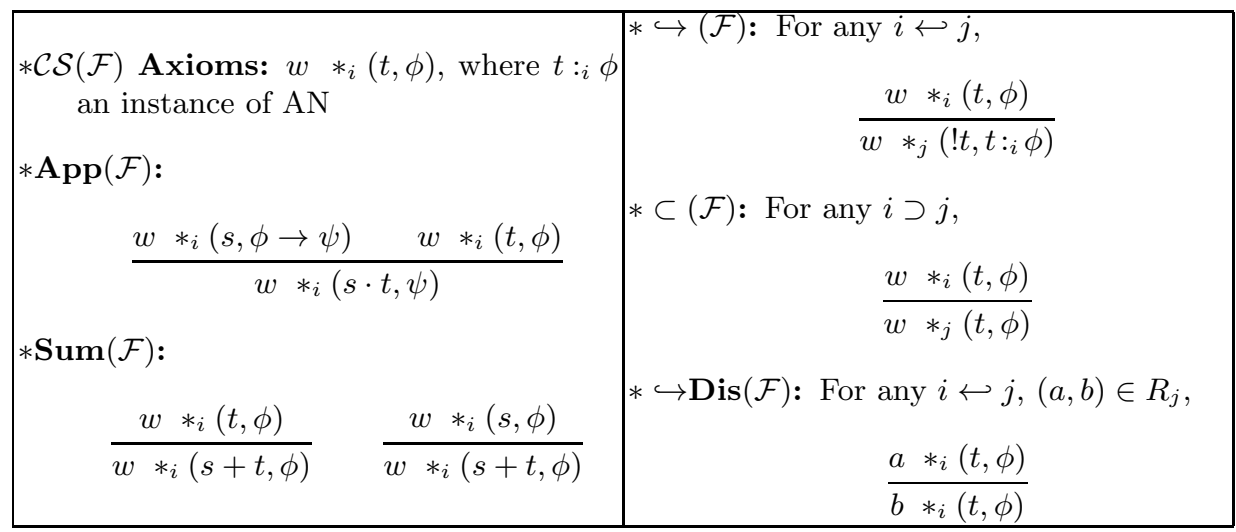

Table 2. The $*^{\mathcal{F}}$-calculus for $J$ : where $\mathcal{F}=\left(W,\left(R_{i}\right)_{i \in N}\right)$ and for every $i \in N$

For $\Phi \subseteq r L_{n}$, the $*$-calculus (without a frame) for $J$ can be defined as $\Phi \vdash_{*} e$ if for every frame $\mathcal{F}$, state $w$ of $\mathcal{F},\{w e \mid e \in * \Phi\} \vdash_{*^{\mathcal{F}}} w *_{i}(t, \phi)$. Notice that for any $v, w$, if $\{w e \mid e \in * \Phi\} \vdash_{*^{\mathcal{F}}} v *_{i}(t, \phi)$, then $\{w e \mid e \in * \Phi\} \vdash_{* \mathcal{F}} w *_{i}(t, \phi)$, therefore the $*$-calculus is the resulting calculus on $*$-expressions after we ignore the frame and world-prefixes (and thus rule $* \hookrightarrow \operatorname{Dis}(\mathcal{F})$ ) in Table 2.3. For an aef $\mathcal{E}$, we write $\mathcal{E} \models w *_{i}(t, \phi)$ when $w \in \mathcal{E}_{i}(t, \phi)$; for set $\Phi$ of $*^{\mathcal{F}}$ - (or $*$-)expressions, $\mathcal{E} \models \Phi$ when $\mathcal{E} \models e$ for every $e \in \Phi$. If $\mathcal{E} \models e$, we may say that $\mathcal{E}$ satisfies $e$.

Proposition 2 ([4], but originally $[11,13])$.

1. Let $\Phi \subseteq r L_{n}$. Then, $* \Phi \vdash_{*}$ e iff for any aef $\mathcal{E} \models * \Phi, \mathcal{E} \models w e$. 
2. For frame $\mathcal{F}$, set of $*^{\mathcal{F}}$-expressions $\Phi, \Phi \vdash_{*} \mathcal{F}$ e iff $\mathcal{E} \models$ e for every aef $\mathcal{E} \models \Phi$.

Proof. For 2, notice that the calculus rules correspond to the closure conditions of the aef, so if $\mathcal{E}_{m} \models e^{6}$ iff $\Phi \vdash_{*_{\mathcal{F}}} e$, then $\mathcal{E}_{m}$ is an aef, so the "if" direction is established; by induction on the calculus derivation, we can also establish for every aef $\mathcal{E}$, if $\mathcal{E}_{m} \models e$, then $\mathcal{E} \models e$. 1 is a direct consequence.

Proposition 3 ([4], but originally $[\mathbf{1 1}, \mathbf{1 3}])$. If $\mathcal{C S} \in \mathrm{P}$ and is schematic, the following problems are in NP:

1. Given a finite frame $\mathcal{F}$, a finite set $S \cup\{e\}$ of $*^{\mathcal{F}}$-expressions, is it the case that $S \vdash_{* \mathcal{F}} e$ ?

2. Given a finite set $S \cup\{e\}$ of $*$-expressions, is it the case that $S \vdash_{*} e$ ?

The shape of a $*$-calculus derivation is mostly described by $t$. We can use $t$ to extract the general shape of the derivation - the term keeps track of the applications of all rules besides $* \subset$ and $* \hookrightarrow$ Dis. We can then plug in to the leaves of the derivation either axioms of the calculus or members of $S$ and unify ( $\mathcal{C S}$ is schematic, so the derivation includes schemes) trying to reach the root. Using Propositions 3 and 1, we can conclude with Corollary 1.

\section{Corollary 1 ([4], but 1 was originally proven in [11]).}

1. If $\mathcal{C S} \in \mathrm{P}$ and is schematic, then deciding for $t:_{i} \phi$ that $J \vdash t:_{i} \phi$ is in NP.

2. If $\mathcal{C S} \in \mathrm{P}$ and is schematic and axiomatically appropriate, then the satisfiability problem for $J$ is in NEXP.

\section{A Universal Lower Bound}

The main result of this section can be found in Theorem 1 and is a lower bound for the complexity of $J$-satisfiability, for an arbitrary multiagent justification $\operatorname{logic} J$, given an axiomatically appropriate, schematic constant specification. We give the theorem first and then its proof.

Theorem 1. If $J$ has an axiomatically appropriate and schematic constant specification, then J-satisfiability is $\Sigma_{2}^{p}$-hard.

Kuznets proved in [12] that, under a schematic constant specification, satisfiability for $\mathrm{J}, \mathrm{JT}, \mathrm{J} 4$, and LP is in $\Sigma_{2}^{p}$ - an upper bound which was also successfully established later for JD [15] and JD4 [1] under the assumption of a schematic and axiomatically appropriate constant specification. In that regard, the lower bound of Theorem 1 is optimal. Kuznets' algorithm is composed of a tableau procedure which analyzes signed formulas of the form $T \phi$, intuitively meaning that $\phi$ is true in the constructed model, and $F \phi$, meaning that $\phi$ is false, with respect to their propositional connectives (and from $T t:_{i} \phi$ gives $T \phi$ in the

${ }^{6} \mathcal{E} \models e$ has only been defined for aefs, but we slightly abuse the notation for convenience. 
presence of Factivity). Eventually it produces formulas of the form $T p, F p$, $T *(t, \phi)$, and $F *(t, \phi)$, where $T *(t, \phi)$ means that the aef of the constructed model makes $(t, \phi)$ true. The tableau process so far takes polynomial time and makes nondeterministic choices to break the propositional connectives and construct a specific branch. Then we need to make sure that there is a model $(\mathcal{E}, \mathcal{V})$ such that $\mathcal{E}(t, \phi)=$ true if $T *(t, \phi)$ is in the branch, $\mathcal{E}(t, \phi)=$ false if $F *(t, \phi)$ is in the branch, $\mathcal{V}(p)=$ true if $T p$ is in the branch, and $\mathcal{V}(p)=$ false if $F p$ is in the branch. The propositional variable part is easy to check - just check that not both $T p$ and $F p$ are in the branch. The aef part is harder to verify, but the branch can give a valid aef if and only if from all $*$-expressions $e$, where $T e$ is in the branch we cannot deduce some $*$-expression $f$ using the $*$-calculus, where $F f$ in the branch. By Proposition 3, this can be verified using an NP-oracle.

The idea behind the reduction we use to prove Theorem 1 is very similar to Milnikel's proof of $\Pi_{2}^{p}$-completeness for J4-provability [17] (which also worked for J-provability). Both Milnikel's and our reduction are from $Q B F_{2}$. The main difference has to do with the way each reduction transforms (or not) the $Q B F$ formula. Milnikel uses the propositional part of the $Q B F$ formula as it is and he introduces existential nondeterministic choices on a satisfiability-testing procedure (think of Kuznets' algorithm as described above) using formulas of the form $x: p \vee y: \neg p$ and universal nondeterministic choices using formulas of the form $x: p \wedge y: \neg p$ and term $[x+y]$ in the final term, forcing a universal choice between $x$ and $y$ during the $*$-calculus testing.

This approach works well for $\mathrm{J}$ and $\mathrm{J} 4$, but it fails in the presence of the Consistency or Factivity axiom, as $x: p \wedge y: \neg p$ becomes inconsistent. For the case of LP, he used a different approach and made use of his assumption of a schematically injective constant specification (i.e. that all constants justify at most one scheme) to construct a term $t$ to specify an intended proof of a formula of the form $\bigwedge_{i}(x: p \wedge y: \neg p) \rightarrow s: \psi$ - which is always provable, since the left part of the implication is inconsistent. In this paper we bypass the problem of the inconsistency of $x: p \wedge y: \neg p$ by replacing each propositional formula by two corresponding propositional variables, $[\chi]^{\top}$ and $[\chi]^{\perp}$ to correspond to " $\chi$ is true" and to " $\chi$ is false" respectively. Therefore, we use $x:[p]^{\top} \wedge y:[p]^{\perp}$ instead of $x: p \wedge y: \neg p$ and we have no inconsistent formulas. As a side-effect we need to use several extra formulas to encode the behavior of the formulas with respect to a truth-assignment - for instance, $[p]^{\top} \rightarrow[p \vee q]^{\top}$ is not a tautology, so we need a formula to assert its truth (see the definitions of $E_{v a l}$ below).

Buss and Kuznets in [8] use the same assumption as Milnikel on the constant specification to give a general lower bound by a reduction from Vertex Cover and a $\Sigma_{2}^{p}$-complete generalization of that problem. Their construction has the advantage that it additionally proves an NP-hardness result for the reflected fragment of the logics they study, while ours does not. On the other hand we do not require a schematically injective constant specification, as, much like Milnikel's construction for $\mathrm{J} 4$, we do not need to limit a $*$-calculus derivation.

Lemma 2 is a simple observation on the resources (number of assumptions) used by a $*$-calculus derivation: if there is a derivation of $*_{i}(t, \phi)$ and $t$ only has 
one appearance of term $s$, then the derivation uses at most one premise of the form $*_{j}(s, \psi)$. In fact, this observation can be generalized to $k$ appearances of $s$ using at most $k$ premises, but this is not important for the proof of Theorem 1 .

Lemma 2. Let $i$ be an agent, $\phi$ a justification formula, $t$ a justification term in which! does not appear, and $s$ a subterm of $t$ which appears at most once in $t$. Let $S_{s}=\left\{s:_{i} \phi_{1}, \ldots, s:_{i} \phi_{k}\right\}$ and $S \subset r L_{n}$, such that $S \cup S_{s}$ is consistent. Then, $S \cup S_{s} \vdash t:_{i} \phi$ if and only if there is some $1 \leq a \leq k$ such that $S \cup\left\{s:_{i} \phi_{a}\right\} \vdash t:_{i} \phi$.

Proof. Easy, by induction on the $*$-calculus derivation (on $t$ ).

The proof of Theorem 1 is by reduction from $Q B F_{2}$, which is the following ( $\Sigma_{2}^{p}$-complete) problem: given a Quantified Boolean Formula,

$$
\phi=\exists x_{1} \exists x_{2} \cdots \exists x_{k} \forall y_{1} \forall y_{2} \cdots \forall y_{k^{\prime}} \psi,
$$

where $\psi$ is a propositional formula on variables $x_{1}, \ldots, x_{k}, y_{1}, \ldots, y_{k^{\prime}}$, is $\phi$ true? That is, are there truth-values for $x_{1}, \ldots, x_{k}$, such that for all truth-values for $y_{1}, \ldots, y_{k^{\prime}}$, a truth-assignment that gives these values makes $\psi$ true?

As mentioned above, for every $\psi_{a} \in \Psi$, let $\left[\psi_{a}\right]^{\top},\left[\psi_{a}\right]^{\perp}$ be new propositional variables. As we argued earlier, we need formulas to help us evaluate the truth of variables under a certain valuation in a way that matches the truth of the original formula, $\psi-[\psi]^{\perp} \rightarrow[\neg \psi]^{\top}$ for instance. These kinds of formulas (prefixed by a corresponding justification term) are gathered into $S(\phi) \cdot T^{J}(\phi)$ is constructed in such a way that under the formulas of $S(\phi)$ and given a valuation $v$

$$
\bigwedge_{v\left(p_{a}\right)=\text { true }} x_{a}:_{i}\left[p_{a}\right]^{\top} \wedge \bigwedge_{v\left(p_{a}\right)=\text { false }} x_{a}:_{i}\left[p_{a}\right]^{\perp} \wedge S(\phi) \vdash T^{J}(\phi):_{i}[\phi]^{\top}
$$

if and only if $v$ makes $\phi$ true. In other words, $T^{J}(\phi)$ encodes the method we would use to evaluate the truth value of $\phi$.

To construct $T^{J}(\phi)$, we first need certain justification terms to encode needed operations to manipulate formulas. We will often need to work on long conjuncts like $\left(\phi_{1} \wedge \cdots \wedge \phi_{r}\right)$, which we can view as a string of formulas. Therefore we need operations like projections $\left(\operatorname{proj}_{x}^{r}\right)$, appending a formula (append), appending a formula to a hypothesis (hypappend), appending the conclusions of two implications (appendconc), and so on. We start by providing these terms.

We define terms $\operatorname{proj}_{x}^{r}$ (for $x \leq r$ ), append, hypappend, and appendconc, to be such that

$$
\begin{gathered}
t:_{i}\left(\phi_{1} \wedge \phi_{2} \wedge \cdots \wedge \phi_{r}\right) \vdash\left[\operatorname{proj}_{x}^{r} \cdot t\right]:_{i} \phi_{x}, \\
t:_{i} \phi_{1}, s:_{i} \phi_{2} \vdash[\text { append } \cdot t \cdot s]:_{i}\left(\phi_{1} \wedge \phi_{2}\right), \\
t:_{i}\left(\phi_{1} \rightarrow \phi_{2}\right) \vdash[\text { hypappend } \cdot t]:_{i}\left(\phi_{1} \rightarrow \phi_{1} \wedge \phi_{2}\right), \text { and } \\
t:_{i}\left(\phi_{1} \rightarrow \phi_{2}\right), s:_{i}\left(\phi_{1} \rightarrow \phi_{3}\right) \vdash[\text { appendconc } \cdot t \cdot s]:_{i}\left(\phi_{1} \rightarrow \phi_{2} \wedge \phi_{3}\right),
\end{gathered}
$$

append, hypappend, and appendconc can simply be any terms such that

$$
\vdash \operatorname{append}_{i}\left(\phi_{1} \rightarrow\left(\phi_{2} \rightarrow \phi_{1} \wedge \phi_{2}\right)\right),
$$




$$
\begin{gathered}
\vdash \text { hypappend: }:_{i}\left(\left(\phi_{1} \rightarrow \phi_{2}\right) \rightarrow\left(\phi_{1} \rightarrow \phi_{1} \wedge \phi_{2}\right)\right) \text {, and } \\
\vdash \text { appendconc: } i\left(\left(\phi_{1} \rightarrow \phi_{2}\right) \rightarrow\left(\left(\phi_{1} \rightarrow \phi_{3}\right) \rightarrow\left(\phi_{1} \rightarrow \phi_{2} \wedge \phi_{3}\right)\right)\right) .
\end{gathered}
$$

Such terms exist, because they justify propositional tautologies and the constant specification is schematic and axiomatically appropriate (see Lemma 1). To define $\operatorname{proj}_{x}^{r}$, we need terms left, right, id,tran, so that

$$
\begin{gathered}
\vdash \text { left: }:_{i}\left(\phi_{1} \wedge \phi_{2} \rightarrow \phi_{1}\right), \quad \vdash \operatorname{right}_{i}\left(\phi_{1} \wedge \phi_{2} \rightarrow \phi_{2}\right), \\
\vdash i d:_{i}\left(\phi_{1} \rightarrow \phi_{1}\right), \text { and } \\
\vdash \operatorname{tran}:_{i}\left(\left(\phi_{1} \rightarrow \phi_{2}\right) \rightarrow\left(\left(\phi_{2} \rightarrow \phi_{3}\right) \rightarrow\left(\phi_{1} \rightarrow \phi_{3}\right)\right) .\right.
\end{gathered}
$$

Again, such terms exist, because they justify propositional tautologies. Then, $\operatorname{proj}_{1}^{1}=i d$; for $r>1, \operatorname{proj}_{r}^{r}=$ right; and for $l<r$, proj $_{l}^{r+1}=\left[\right.$ trans $_{\text {left }} \cdot$ proj $\left._{l}^{r}\right]$.

Now we provide the formulas that will help us with evaluating the truth of the propositional part of the QBF formula under a valuation. These were axioms provided by the constant specification in Milnikel's proof [17], but as we argued before, we need the following formulas in our case. Let $\Psi=\left\{\psi_{1}, \ldots, \psi_{l}\right\}$ be an ordering of all subformulas of $\psi$, such that if $a<b$, then $\left|\psi_{a}\right| \leq\left|\psi_{b}\right|^{7}$. Furthermore, $\rho=|\{\chi \in \Psi|| \chi \mid=1\}|$ and for every $1 \leq j \leq l$,

if $\psi_{j}=\neg \gamma$, then Eval $_{j}=\operatorname{truth}_{j}: i\left([\gamma]^{\top} \rightarrow\left[\psi_{j}\right]^{\perp}\right) \wedge \operatorname{truth}_{j}: i\left([\gamma]^{\perp} \rightarrow\left[\psi_{j}\right]^{\top}\right)$; if $\psi_{j}=\gamma \vee \delta$, then

$$
\begin{gathered}
\text { Eval }_{j}=\text { truth }_{j}:_{i}\left([\gamma]^{\top} \wedge[\delta]^{\top} \rightarrow\left[\psi_{j}\right]^{\top}\right) \wedge \text { truth }_{j}:_{i}\left([\gamma]^{\top} \wedge[\delta]^{\perp} \rightarrow\left[\psi_{j}\right]^{\top}\right) \\
\wedge \text { truth }_{j}: i\left([\gamma]^{\perp} \wedge[\delta]^{\top} \rightarrow\left[\psi_{j}\right]^{\top}\right) \wedge \text { truth }_{j}:{ }_{i}\left([\gamma]^{\perp} \wedge[\delta]^{\perp} \rightarrow\left[\psi_{j}\right]^{\perp}\right)
\end{gathered}
$$

if $\psi_{j}=\gamma \wedge \delta$, then

$$
\begin{gathered}
\text { Eval }_{j}=\text { truth }_{j}:_{i}\left([\gamma]^{\top} \wedge[\delta]^{\top} \rightarrow\left[\psi_{j}\right]^{\top}\right) \wedge \text { truth }_{j}: i\left([\gamma]^{\top} \wedge[\delta]^{\perp} \rightarrow\left[\psi_{j}\right]^{\perp}\right) \\
\wedge \text { truth }_{j}:_{i}\left([\gamma]^{\perp} \wedge[\delta]^{\top} \rightarrow\left[\psi_{j}\right]^{\perp}\right) \wedge \text { truth }_{j}:_{i}\left([\gamma]^{\perp} \wedge[\delta]^{\perp} \rightarrow\left[\psi_{j}\right]^{\perp}\right)
\end{gathered}
$$

if $\psi_{j}=\gamma \rightarrow \delta$, then

$$
\begin{gathered}
\text { Eval }_{j}=\text { truth }_{j}:_{i}\left([\gamma]^{\top} \wedge[\delta]^{\top} \rightarrow\left[\psi_{j}\right]^{\top}\right) \wedge \text { truth }_{j}: i\left([\gamma]^{\top} \wedge[\delta]^{\perp} \rightarrow\left[\psi_{j}\right]^{\perp}\right) \\
\wedge \text { truth }_{j}: i\left([\gamma]^{\perp} \wedge[\delta]^{\top} \rightarrow\left[\psi_{j}\right]^{\top}\right) \wedge \text { truth }_{j}:{ }_{i}\left([\gamma]^{\perp} \wedge[\delta]^{\perp} \rightarrow\left[\psi_{j}\right]^{\top}\right) .
\end{gathered}
$$

\footnotetext{
$\overline{7 \text { assume a }|\cdot|}$, such that $\left|p_{j}\right|=1$ and if $\gamma$ is a proper subformula of $\delta$, then $|\gamma|<|\delta|$
} 
We now construct term $T^{J}(\phi)$. To do this we first construct terms $T^{a}$, where $1 \leq a \leq l$. Given a valuation $v$ in the form $x_{1}:_{i}\left[p_{1}\right]^{v_{1}}, \ldots, x_{k}:_{i}\left[p_{k}\right]^{v_{k}}, T^{1}$ through $T^{k}$ simply gather these formulas in one large conjunct (or string). Then for $k+1 \leq a \leq l, T^{a}$ evaluates the truth of $\psi_{a}$, resulting in either $[\psi]^{\top}$ or $[\psi]^{\perp}$ and appending the result at the end of the conjunct.

Let $T^{1}=x_{1}$ and for every $1<a \leq k, T^{a}=\left[\right.$ append $\left.\cdot T^{a-1} \cdot x_{a}\right]$. It is not hard to see that for $v_{1}, \ldots, v_{k} \in\{\top, \perp\}$,

$$
x_{1}:_{i}\left[p_{1}\right]^{v_{1}}, \ldots, x_{k}:_{i}\left[p_{k}\right]^{v_{k}} \vdash T^{k}:_{i}\left(\left[p_{1}\right]^{v_{1}} \wedge \cdots \wedge\left[p_{k}\right]^{v_{k}}\right) .
$$

If $\psi_{a}=\neg \psi_{b}$, then

$$
T^{a}=\text { hypappend } \cdot\left[\text { trans } \cdot \operatorname{proj}_{b}^{a-1} \cdot \operatorname{truth}_{a}\right] \cdot T^{a-1} \text { and }
$$

if $\psi_{a}=\psi_{b} \circ \psi_{c}$, then

$$
T^{a}=\text { hypappend } \cdot\left[\text { trans } \cdot\left[\text { appendconc } \cdot \operatorname{proj}_{b}^{a-1} \cdot \operatorname{proj}_{c}^{a-1}\right] \cdot \operatorname{truth}_{a}\right] \cdot T^{a-1} .
$$

Let

$$
S(\phi)=\bigwedge_{\rho<j \leq l} \operatorname{Eval}_{j}
$$

and given a truth valuation $v$, let

$$
S^{v}(\phi)=\bigwedge_{v\left(p_{j}\right)=\text { true }} x_{j}:_{i}\left[p_{j}\right]^{\top} \wedge \bigwedge_{v\left(p_{j}\right)=\text { false }} x_{j}:_{i}\left[p_{j}\right]^{\perp} \wedge \bigwedge_{\rho<j \leq l} E v a l_{j} .
$$

By induction on $a$, for every truth assignment $v$,

$$
S^{v}(\phi) \vdash T^{a}:_{i}\left(\left[\psi_{1}\right]^{v_{1}} \wedge \cdots \wedge\left[\psi_{a}\right]^{v_{a}}\right),
$$

where if $\psi_{b}$ is true under $v$, then $v_{b}=\top$ and $v_{b}=\perp$ otherwise. The cases where $a \leq k$ are easy to see from (1). For the remaining cases it is enough to demonstrate that

if $\psi_{a}=\neg \psi_{j}$, then $S(\phi) \vdash\left[\right.$ trans $\cdot \operatorname{proj}_{j}^{a-1} \cdot$ truth $\left._{a} \cdot T^{a-1}\right]:_{i}\left[\psi_{a}\right]^{v_{a}}$ and if $\psi_{a}=\psi_{b} \circ \psi_{c}$, then

$$
S(\phi) \vdash\left[\text { trans } \cdot\left[\text { appendconc } \cdot \operatorname{proj}_{b}^{a-1} \cdot \operatorname{proj}_{c}^{a-1}\right] \cdot \text { truth }_{a} \cdot T^{a-1}\right]: i\left[\psi_{a}\right]^{v_{a}},
$$

which is not hard to see by the way we designed each term.

Finally, let $T^{J}(\phi)=\left[\right.$ right $\left.\cdot T^{l}\right]$. We can now prove Lemma 3:

Lemma 3. For every $n \in \mathbb{N}$ and agent $i \in N, T^{J}(\phi), S(\phi)$ are computable in polynomial time with respect to $|\phi| . \phi$ is true under truth assignment $v$ if and only if

$$
\bigwedge_{v\left(p_{a}\right)=\text { true }} x_{a}:_{i}\left[p_{a}\right]^{\top} \wedge \bigwedge_{v\left(p_{a}\right)=\text { false }} x_{a}:_{i}\left[p_{a}\right]^{\perp} \wedge S(\phi) \vdash T^{J}(\phi):_{i}[\phi]^{\top} .
$$


Proof. From the above construction we can see that if $\phi$ is true under $v$ then $S^{v}(\phi) \vdash T^{J}(\phi):_{i}[\phi]^{\top}$. On the other hand, if $S^{v}(\phi) \vdash T^{J}(\phi):_{i}[\phi]^{\top}$, then $* S^{v}(\phi) \vdash_{*} *_{i}\left(\left[\right.\right.$ right $\left.\left.\cdot T^{l}\right],[\phi]^{\top}\right)$, which in turn gives $\left(S^{v}(\phi)\right)^{\#_{i}} \vdash[\phi]^{\top}$ (the terms do not include the operator ! and thus the right side of a $*$-derivation is a derivation in propositional logic). If $\phi$ is not true under $v$, then let $v^{\prime}$ be the valuation, such that $v^{\prime}\left([\psi]^{\top}\right)=$ true iff $\psi$ is true under $v$ and $v^{\prime}\left([\psi]^{\perp}\right)=$ true iff $\psi$ is false under $v$. Then all of $\left(S^{v}(\phi)\right)^{\#_{i}}$ is true under $v^{\prime}$ and $[\phi]^{\top}$ is not, therefore $\left(S^{v}(\phi)\right)^{\#_{i}} \forall[\phi]^{\top}$, so $S^{v}(\phi) \forall T^{J}(\phi):_{i}[\phi]^{\top}$.

Corollary 2. The $Q B F$ formula $\exists p_{1}, \ldots, p_{k} \forall p_{k+1}, \ldots, p_{k+l} \phi$ is true if and only if the following formula is $J$-satisfiable:

$\bigwedge_{j=1}^{k}\left(x_{j}:{ }_{i}\left[p_{j}\right]^{\top} \vee x_{j}:_{i}\left[p_{j}\right]^{\perp}\right) \wedge \bigwedge_{j=k+1}^{l}\left(x_{j}:_{i}\left[p_{j}\right]^{\top} \wedge x_{j}:_{i}\left[p_{j}\right]^{\perp}\right) \wedge S(\neg \phi) \wedge \neg T^{J}(\neg \phi)[\neg \phi]^{\top}$.

Proof. If

$\bigwedge_{j=1}^{k}\left(x_{j}: i\left[p_{j}\right]^{\top} \vee x_{j}: i\left[p_{j}\right]^{\perp}\right) \wedge \bigwedge_{j=k+1}^{l}\left(x_{j}: i\left[p_{j}\right]^{\top} \wedge x_{j}: i\left[p_{j}\right]^{\perp}\right) \wedge S(\neg \phi) \wedge \neg T^{J}(\neg \phi)[\neg \phi]^{\top}$

is not satisfiable, then

$\bigwedge_{j=1}^{k}\left(x_{j: i}\left[p_{j}\right]^{\top} \vee x_{j}: i\left[p_{j}\right]^{\perp}\right) \wedge \bigwedge_{j=k+1}^{l}\left(x_{j}: i\left[p_{j}\right]^{\top} \wedge x_{j}: i\left[p_{j}\right]^{\perp}\right) \wedge S(\neg \phi) \vdash T^{J}(\neg \phi)[\neg \phi]^{\top}$,

and then for every choice $c_{1}:\{1, \ldots, k\} \longrightarrow\{\top, \perp\}$,

$$
\bigwedge_{j=1}^{k}\left(x_{j}:_{i}\left[p_{j}\right]^{c_{1}(j)}\right) \wedge \bigwedge_{j=k+1}^{l}\left(x_{j}:_{i}\left[p_{j}\right]^{\top} \wedge x_{j}:_{i}\left[p_{j}\right]^{\perp}\right) \wedge S(\neg \phi) \vdash T^{J}(\neg \phi)[\neg \phi]^{\top},
$$

and then since every variable from $x_{1}, \ldots, x_{k+l}$ appears at most once in $T^{J}$ and $T^{J}$ does not include !, by Lemma 2 there is some choice $c_{2}:\{1, \ldots, l\} \longrightarrow\{\top, \perp\}$ such that

$$
\bigwedge_{j=1}^{k}\left(x_{j}:_{i}\left[p_{j}\right]^{c_{1}(j)}\right) \wedge \bigwedge_{j=k+1}^{l}\left(x_{j}:_{i}\left[p_{j}\right]^{c_{2}(j)}\right) \wedge S(\neg \phi) \vdash T^{J}(\neg \phi)[\neg \phi]^{\top} .
$$

Therefore, for every assignment of truth-values on $p_{1}, \ldots, p_{k}$ there truth-values for $p_{k+1}, \ldots, p_{l+k}$ that make $\phi$ false.

On the other hand, if

$$
\bigwedge_{j=1}^{k}\left(x_{j: i}\left[p_{j}\right]^{\top} \vee x_{j}: i\left[p_{j}\right]^{\perp}\right) \wedge \bigwedge_{j=k+1}^{l}\left(x_{j}:_{i}\left[p_{j}\right]^{\top} \wedge x_{j}:_{i}\left[p_{j}\right]^{\perp}\right) \wedge S(\neg \phi) \wedge \neg T^{J}(\neg \phi)[\neg \phi]^{\top}
$$


is satisfiable, then there is some choice $c_{1}:\{1, \ldots, k\} \longrightarrow\{\top, \perp\}$, such that

$$
\bigwedge_{j=1}^{k}\left(x_{j}:_{i}\left[p_{j}\right]^{c_{1}(j)}\right) \wedge \bigwedge_{j=k+1}^{l}\left(x_{j}:_{i}\left[p_{j}\right]^{\top} \wedge x_{j}:_{i}\left[p_{j}\right]^{\perp}\right) \wedge S(\neg \phi) \wedge \neg T^{J}(\neg \phi)[\neg \phi]^{\top}
$$

is satisfiable, and then since every variable from $x_{1}, \ldots, x_{k+l}$ appears at most once in $T^{J}$, for every choice $c_{2}:\{1, \ldots, l\} \longrightarrow\{\top, \perp\}$,

$$
\bigwedge_{j=1}^{k}\left(x_{j}:_{i}\left[p_{j}\right]^{c_{1}(j)}\right) \wedge \bigwedge_{j=k+1}^{l}\left(x_{j}:_{i}\left[p_{j}\right]^{c_{2}(j)}\right) \wedge S(\neg \phi) \forall T^{J}(\neg \phi)[\neg \phi]^{\top} .
$$

Therefore, there is some truth assignment on $p_{1}, \ldots, p_{k}$ such that every truth assignment on $p_{k+1}, \ldots, p_{l+k}$ makes $\phi$ true.

Theorem 1 is then a direct corollary of the above.

\section{A NEXP-complete Justification Logic}

The justification logic we prove to have a NEXP-complete satisfiability problem is the 4-agent logic $J_{H}=(4, \subset, \hookrightarrow, F)_{\mathcal{C S}}$, where

$-\subset=\{(3,4)\}$,

$-\hookleftarrow=\{(1,2),(2,3),(4,4)\}$,

$-F(1)=F(2)=\mathrm{J}, F(3)=F(4)=\mathrm{JD}$, and

$-\mathcal{C S}$ is any axiomatically appropriate and schematic constant specification.

The agents of $J_{H}$ are based on justification logics J and JD - and essentially JD4, as agent 4 has Positive Introspection. Agent 3 has a significant variety of justifications. Since $1 \hookleftarrow 2 \hookleftarrow 3,3$ is aware of the justifications of 2 , who in turn is aware of the justifications of 1 . Therefore, 3 can simulate the reasoning of 2 who can simulate the reasoning of 1 . Additionally, 3 accepts two types of justifications: the ones 3 receives from 4, which come with Positive Introspection and the other ones 3 accepts, which do not. As Theorem 2 demonstrates, this complex interaction among agent 3's justifications results in the significant hardness of $J_{H}$-satisfiability.

If we only focus on agents 3 and 4, we have a PSPACE-complete justification logic $[3,4]$. In a tableau procedure which constructs a model for a given formula (like the one in [4]), this means that we may have to consider a large number of states. If we could simply explore smaller parts of the model as we can often do for Modal Logic, we could still end up with an (alternating perhaps) polynomial space algorithm. The satisfiability-testing procedures for Justification Logic have another part, though, and that is testing whether certain $*^{\mathcal{F}}$-expressions can be derived in a frame $\mathcal{F}$ from a certain set of $*^{\mathcal{F}}$-expressions using the $*$-calculus which corresponds to asking whether there is an aef that satisfies certain expressions and not others. By Proposition 3, this can be done using a nondeterministic procedure which takes time polynomial with respect to $|\mathcal{F}|$ and to the overall 
size of the set of $*^{\mathcal{F}}$-expressions. Although the complexity of that procedure is not something which increases the overall complexity of satisfiability-testing [4], to run it we must keep the whole frame $\mathcal{F}$ in memory and $\mathcal{F}$ can be large, which requires exponential time and more than polynomial space. Nondeterminism is introduced as we apply the tableau rules, as some require nondeterministic choices. Assuming PSPACE $\neq$ NEXP, this is a difficulty we cannot overcome.

Theorem 2. $J_{H}$-satisfiability is NEXP-hard.

The reduction we use is from a subproblem of the SCHÖNFINKEL-BERNAYS SAT problem, which we call BINARY SCHÖNFINKEL-BERNAYS SAT:

Given a first-order formula $\phi$ of the form $\exists x_{1} \cdots \exists x_{k} \forall y_{1} \cdots \forall y_{k^{\prime}} \psi$, where $\psi$ contains no quantifiers or function symbols, is $\phi$ satisfiable by a firstorder model of exactly two elements?

The general SCHÖNFINKEL-BERNAYS SAT problem does not require that a satisfying model has exactly two elements and is known to be NEXP-complete [16]; BINARY SCHÖNFINKEL-BERNAYS SAT remains NEXP-complete.

The reduction for Theorem 2 is essentially an extended version of the reduction we used to prove Theorem 1. Like then, consider a construction of a satisfying model, only this time it is an F-model with several states and accessibility relations for agents. Another difference is, of course, that now the original formula is from the first-order language. However, in the BINARY SCHÖNFINKEL$B E R N A Y S$ SAT formulation, each (first-order) variable is quantified over two possible values (the elements of the two-element model), so they are essentially propositional variables. Since this is satisfiability we must existentially quantify each relation symbol over all $2^{r+1} r$-ary relations. We can encode such a nondeterministic choice by forcing the existence of an exponential number of states, each representing one $r$-tuple $v=v_{1}, \ldots, v_{r}$ of the two possible values 0 and 1 (as mentioned above, we can do this using agents 3 and 4) by having var: $:_{1}\left[p_{a}\right]^{v_{a}}$ being true and then at each such state enforce the choice between $r e:_{1}[R]^{\top}$ and rel $:_{1}[R]^{\perp}$, meaning that $v \in R$ or $v \notin R$ respectively - where $R$ an actual relation. In such a state conjunctions of the form gather $:_{1}\left(\left[p_{1}\right]^{v_{1}} \wedge \cdots \wedge\left[p_{r}\right]^{v_{r}} \wedge[R]^{\triangle}\right)$ (where $\triangle=\top$ or $\perp$ ) encode this choice. Due to the particular interaction among the agents and the logics they are based on, in the constructed model gather $:_{1}\left(\left[p_{1}\right]^{v_{1}} \wedge \cdots \wedge\left[p_{r}\right]^{v_{r}} \wedge[R]^{\triangle}\right)$ is true in a state if and only if that state represents $v$ and $\triangle=\top$ iff $v \in R$. Already this $J_{H}$-model encodes a first-order model. The trick now is to be able to gather in one state all these formulas that encode the relations through the aef closure conditions (i.e. through the $*$-calculus), but making sure that individual conjuncts (i.e. something of the form var: $:_{1}[p]^{\triangle}$ or rel $:_{1}[R]^{\triangle}$ ) cannot be also transfered to that state through the calculus - in that case we would be able to construct gather $:_{1}\left(\left[p_{1}\right]^{v_{1}} \wedge \cdots \wedge\left[p_{r}\right]^{v_{r}} \wedge[R]^{\triangle}\right)$ for additional, invalid combinations of $(v, \triangle)$. This is achieved by considering formulas of the form ! gather $:_{2}$ gather $:_{1}\left(\left[p_{1}\right]^{v_{1}} \wedge \cdots \wedge\left[p_{r}\right]^{v_{r}} \wedge[R]^{\triangle}\right)$. The constructed model has empty accessibility relations for agents 1 and 2, thus such formulas can move freely through the accessibility relation of agent 3 (since 
$2 \hookleftarrow 3$ and because of Distribution), but this is not the case for anything of the form $t:_{1} \chi$ (since $1 \nLeftarrow 3,4$ ). Using certain additional formulas we can make sure that ! gather $:_{2}$ gather $:_{1}\left(\left[p_{1}\right]^{v_{1}} \wedge \cdots \wedge\left[p_{r}\right]^{v_{r}} \wedge[R]^{\triangle}\right) \rightarrow\left[R\left(x_{1}, \ldots, x_{r}\right)\right]^{\triangle}$ becomes true if and only if $x_{1}, \ldots, x_{r}$ are interpreted as $v_{1}, \ldots, v_{r}$. The remaining of the formulas and methods we use are very similar to the ones we use for Theorem 1.

By combining Corollary 1 and Theorem 2, we can claim the following:

Corollary 3. $J_{H}$-satisfiability is NEXP-complete.

\subsection{Proof of Theorem 2}

The reduction we use is from (a variation of) the SCHÖNFINKEL-BERNAYS SAT problem: given a first-order formula $\phi$ of the form

$$
\exists x_{1} \cdots \exists x_{k} \forall y_{1} \cdots \forall y_{k^{\prime}} \psi
$$

where $\psi$ contains no quantifiers or function symbols, is $\phi$ satisfiable by a firstorder model?

SCHÖNFINKEL-BERNAYS SAT is known to be NEXP-complete ([16]). Furthermore, it is not hard to see that if

$$
\exists x_{1} \cdots \exists x_{k} \forall y_{1} \cdots \forall y_{k^{\prime}} \psi
$$

is satisfiable, then it is satisfiable by a model of at most $k$ elements. For the coming reduction, we instead use for convenience a simplified version of this problem, which we call BINARY SCHÖNFINKEL-BERNAYS SAT and is the same problem, only instead we ask if $\exists x_{1} \cdots \exists x_{k} \forall y_{1} \cdots \forall y_{k^{\prime}} \psi$ is satisfiable by a first-order model of exactly two elements.

For the reductions that follow we use the following notation: for a nonnegative integer $x \in \mathbb{N}$, let $\operatorname{bin}(x)=\operatorname{bin}_{0}(g), \ldots, \operatorname{bin}_{\log g}(g)$ be its binary representation. Furthermore, like in Section 3, for every propositional and first-order formula $\psi$ we introduce propositional variables $[\psi]^{\top}$ and $[\psi]^{\perp}$.

Lemma 4. BINARY SCHÖNFINKEL-BERNAYS SAT is NEXP-complete.

Proof. Let $\phi$ be a first-order formula of the form

$$
\exists x_{1} \cdots \exists x_{k} \forall y_{1} \cdots \forall y_{k^{\prime}} \psi
$$

where $\psi$ contains no quantifiers or function symbols. Furthermore, we assume that $\psi$ contains no constants. We can replace each $x_{a}$ by $\boldsymbol{x}_{a}=x_{a}^{1}, x_{a}^{2}, \ldots, x_{a}^{\lceil\log k\rceil}$ and each $y_{b}$ by $\boldsymbol{y}_{b}=y_{b}^{1}, y_{b}^{2}, \ldots, y_{b}^{\lceil\log k\rceil}$ in the quantifiers and wherever they appear in a relation. Therefore $\exists x_{a}$ is replaced by $\exists x_{a}^{1} \exists x_{a}^{2} \cdots \exists x_{a}^{\lceil\log k\rceil}$ ( $\exists \boldsymbol{x}_{\boldsymbol{a}}$ for short) and $\forall x_{a}$ is replaced by $\forall x_{a}^{1} \forall x_{a}^{2} \cdots \forall x_{a}^{\lceil\log k\rceil}$ ( $\forall \boldsymbol{y}_{\boldsymbol{a}}$ for short) and $R\left(z_{1}, \ldots, z_{r}\right)$ is replaced by $R\left(\boldsymbol{z}_{1}, \ldots, \boldsymbol{z}_{r}\right)$. Furthermore, every expression $z=z^{\prime}$ where $z, z^{\prime}$ 
are variables, is replaced by $\bigwedge_{1 \leq a \leq\lceil\log k\rceil} z^{a}=z^{a}\left(\boldsymbol{z}=\boldsymbol{z}^{\prime}\right.$ for short). The result of all these replacements in $\psi$ is called $\psi^{\prime}$. The new formula is:

$$
\phi^{\prime}=\exists \boldsymbol{x}_{1} \cdots \exists \boldsymbol{x}_{k} \forall \boldsymbol{y}_{1} \cdots \forall \boldsymbol{y}_{k^{\prime}}\left(\bigwedge_{b=1}^{k^{\prime}} \bigvee_{a=1}^{k} \boldsymbol{x}_{\boldsymbol{a}}=\boldsymbol{y}_{\boldsymbol{b}} \rightarrow \psi^{\prime}\right)
$$

We can also define a corresponding transformation of first-order models: assume that the universe of model $\mathcal{M}$ for $\phi$ is a set of at most $k$ natural numbers (each of which is at most $k-1$ and an interpretation for some $x_{a}$ ); then $\mathcal{M}^{\prime}$ is the model with $\{0,1\}$ as its universe, where for every relation $R$ (on tuples of naturals) of $\mathcal{M}$ there is some $R^{\prime}$, which is essentially the same relation, but on the binary representations of the elements of $\mathcal{M}$. That is,

$$
R^{\prime}=\left\{\left(\operatorname{bin}\left(a_{1}\right), \ldots, \operatorname{bin}\left(a_{r}\right)\right) \in\{0,1\}^{*} \mid\left(a_{1}, \ldots, a_{r}\right) \in R\right\}
$$

It is not hard to see that if $\mathcal{M}$ satisfies the original formula, then $\mathcal{M}^{\prime}$ satisfies the new one: each $\boldsymbol{x}_{a}$ can be interpreted as the binary representation of the interpretation of $x_{a}$ in $\mathcal{M}$ and notice that the added equality assertions effectively limit the $\boldsymbol{y}$ 's to range over the interpretations of the $\boldsymbol{x}$ 's, which are then exactly the image of the elements of $\mathcal{M}$.

On the other hand, if $\phi^{\prime}$ is satisfied by a model with $\{0,1\}$ as its universe, then $\phi$ is satisfied by the model which has the $\lceil\log k\rceil$-tuples of $\{0,1\}$ that are the interpretations of $\boldsymbol{x}_{1}, \ldots, \boldsymbol{x}_{k}$ as elements and as relations the restrictions of the two-element model's relations on these tuples.

Given a first-order formula $\phi$ as above, we construct a justification formula, $\phi^{J}$, in polynomial time, such that $\phi$ is satisfiable by a two-element model if and only if $\phi$ is satisfiable by a $J$-model. The reader will notice several similarities to the proof of Theorem 1 .

Let

$$
\phi=\exists x_{1} \cdots \exists x_{k} \forall y_{1} \cdots \forall y_{k^{\prime}} \psi
$$

be such a formula, where $\psi$ contains no quantifiers or function symbols. Let $R_{1}, \ldots, R_{m}$ be the relation symbols appearing in $\psi, a_{1}, \ldots, a_{m}$ their respective arities. Then, let $\alpha=\left\{i \in \mathbb{N} \mid \exists r \leq m\right.$ s.t. $\left.i \leq a_{r}\right\}$; then, $|\alpha|=\max \left\{a_{1}, \ldots, a_{m}\right\}$. We also define: $X=\left\{x_{1}, \ldots, x_{k}\right\} ; Y=\left\{y_{1}, \ldots, y_{k^{\prime}}\right\} ; Z=X \cup Y ; \rho_{0}=k+k^{\prime}$.

For this reduction, in addition to the terms introduced in Section 3, we define the following justification terms. If we expect a term to justify a tautological scheme of fixed length, then we can just assume the term exists and has some constant size. Otherwise we construct the term in a way that gives it size polynomial with respect to the formula it (provably) justifies. Again we need certain terms to encode manipulations of long conjunctions (which we can see as strings) and we start with these.

addhyp is such that $\vdash$ addhyp: $:_{1}(\phi \rightarrow(\psi \rightarrow \phi))$;

replaceleft is such that $\vdash$ replaceleft $:_{1}\left(\left(\phi \rightarrow \phi^{\prime}\right) \rightarrow\left((\phi \wedge \psi) \rightarrow\left(\phi^{\prime} \wedge \psi\right)\right)\right)$, while 
replaceright is such that $\vdash$ replaceright: $:_{1}\left(\left(\psi \rightarrow \psi^{\prime}\right) \rightarrow\left((\phi \wedge \psi) \rightarrow\left(\phi \wedge \psi^{\prime}\right)\right)\right)$; We define replace ${ }_{l}^{k}$ in the following way:

$$
\text { replace }_{k}^{k}=\text { replaceright, }
$$

while for $l<k$,

$$
\text { replace }_{l}^{k}=\text { trans } \cdot \text { replace } e_{l}^{k-1} \cdot \text { replaceleft } .
$$

Then it is not hard to see by induction on $k-l$ that

$$
\vdash \text { replace }:_{l}:_{1}\left(\left(\phi_{l} \rightarrow \phi_{l}^{\prime}\right) \rightarrow\left(\left(\phi_{1} \wedge \cdots \wedge \phi_{l} \wedge \cdots \wedge \phi_{k}\right) \rightarrow\left(\phi_{1} \wedge \cdots \wedge \phi_{l}^{\prime} \wedge \cdots \wedge \phi_{k}\right)\right)\right) .
$$

We define mphypoth to be such that

$$
\vdash \text { mphypoth: }:_{1}((\phi \rightarrow \psi) \rightarrow((\phi \rightarrow(\psi \rightarrow \chi)) \rightarrow(\phi \rightarrow \chi))) .
$$

We use justification variables $\operatorname{var}_{1}, \ldots, v a r_{a_{r}}$, rel $_{r}$ for every $r \in[m]$. For $1 \leq r \leq m$ we define gather $_{r}$ in the following way:

$$
\text { gather }_{r}=\left[\text { append } \cdot\left[\text { append } \cdots\left[\text { append } \cdot \text { var }_{1}\right] \cdots \text { var }_{a_{r}}\right] \cdot \text { rel }_{r}\right] \text {, }
$$

For every $1 \leq j \leq a_{r}+1$, let $v_{j}, v_{j}^{\prime} \in\{\top, \perp\}$. Then, for propositional variables $p_{1}, \ldots, p_{a_{r}}$,

$$
\bigwedge_{j=1}^{a_{r}} \operatorname{var}_{j}:_{1}\left[p_{j}\right]^{v_{j}} \wedge r e l_{r}:_{1}\left[R_{r}\right]^{v_{a_{r}+1}} \vdash \text { gather }_{r}:_{1}\left(\left[p_{1}\right]^{v_{1}^{\prime}} \wedge \cdots \wedge\left[p_{a_{r}}\right]^{v_{a_{r}}^{\prime}} \wedge\left[R_{r}\right]^{v_{a_{r}+1}^{\prime}}\right)
$$

if and only if for every $1 \leq j \leq a_{r}+1, v_{j}=v_{j}^{\prime}$ (see the proof of Lemma 3 ). In fact it is not hard to see that if

$$
\bigwedge_{j=1}^{a_{r}} \operatorname{var}_{j}:_{1}\left[p_{j}\right]^{v_{j}} \wedge \operatorname{rel}_{r}:_{1}\left[R_{r}\right]^{v_{a_{r}+1}} \vdash \text { gather }_{r}: 1 \chi,
$$

then $\bigwedge_{j=1}^{a_{r}}\left[p_{j}\right]^{v_{j}} \wedge\left[R_{r}\right]^{v_{a_{r}+1}} \vdash \chi$ : operator ! does not appear in gather $_{r}$, so the right-hand side of a corresponding $*$-calculus derivation for $*_{1}\left(\right.$ gather $\left._{r}, \chi\right)$ is a propositional derivation of $\chi$ from $\left[p_{1}\right]^{v_{1}}, \ldots,\left[p_{a_{r}}\right]^{v_{a_{r}},\left[R_{r}\right]^{v_{a_{r}+1}} \text { and some }}$ propositional tautologies.

To give some intuition, conjunction $\bigwedge_{j=1}^{a_{r}} \operatorname{var}_{j}:_{1}\left[p_{j}\right]^{v_{j}} \wedge \operatorname{rel}_{r}:_{1}\left[R_{r}\right]^{v_{a_{r}+1}}$ means that $\left(v_{1}, \ldots, v_{a_{r}}\right) \in R_{r}$ in a corresponding first-order model.

We use justification variables value $z$ and match $\left(z, p_{l}\right)$ for all $z \in Z, l \in \alpha$. For every $z \in X$, we define $V_{z}=$ value $_{z}:_{1}[z]^{\top} \vee$ value $_{z}:_{1}[z]^{\perp}$; for every $z \in Y, V_{z}=$ value $_{z}:_{1}[z]^{\top} \wedge$ value $_{z}:_{1}[z]^{\perp}$.

We also define

$$
\text { Match }=\bigwedge_{\substack{l \in \alpha \\ z \in Z \\ \triangle \in\{\top, \perp\}}} \operatorname{match}\left(z, p_{l}\right):_{1}\left([z]^{\triangle} \rightarrow\left(\left[p_{l}\right]^{\triangle} \rightarrow o k_{l}\right)\right)
$$


For every $R_{r}(z)$ which appears in $\psi$ and $0 \leq b \leq a_{r}$, we define $\operatorname{match}_{b}^{R_{r}(z)}$ in the following way: match $_{0}^{R_{r}(\boldsymbol{z})}=$ addhyp $_{\text {gather }}$ and if $b>0$ and $z_{b}=x_{l}$ or $z_{b}=y_{l-k}$, then match $_{b}^{R_{r}(\boldsymbol{z})}$ is defined to be the term $\left[\right.$ mphypoth $\left.\cdot \operatorname{match}_{b-1}^{R_{r}(\boldsymbol{z})} \cdot\left[\operatorname{tran} \cdot\left[\operatorname{tran} \cdot \operatorname{project}_{l}^{\rho_{1}} \cdot \operatorname{match}\left(z_{b}, b\right)\right] \cdot \operatorname{replace}_{b}^{a_{r}+1}\right]\right]$.

We can see by induction on $b$ that for every $0 \leq b \leq a_{r}$,

Match, gather $:_{1}\left(\left[p_{1}\right]^{v_{1}^{\prime}} \wedge \cdots \wedge\left[p_{a_{r}}\right]^{v_{a_{r}}^{\prime}} \wedge\left[R_{r}\right]^{v_{a_{r}+1}}\right) \vdash$

$$
\begin{array}{r}
\vdash \operatorname{match}_{b}^{R_{r}\left(z_{1}, \ldots, z_{\left.a_{r}\right)}:_{1}\right.}\left(\left(\left[x_{1}\right]^{v_{1}} \wedge \cdots \wedge\left[x_{k}\right]^{v_{k}} \wedge\left[y_{1}\right]^{v_{k+1}} \wedge \cdots \wedge\left[y_{k^{\prime}}\right]^{v_{k^{\prime}+k}}\right) \rightarrow\right. \\
\left.\rightarrow\left(o k_{1} \wedge \cdots \wedge o k_{b} \wedge\left[p_{b+1}\right]^{v_{b+1}^{\prime}} \wedge \cdots \wedge\left[p_{a_{r}}\right]^{v_{a_{r}}^{\prime}} \wedge\left[R_{r}\right]^{v_{a_{r}+1}}\right)\right)
\end{array}
$$

if and only if for every $j \in\left[a_{r}\right]$ and $j^{\prime} \in\left[k+k^{\prime}\right]$, if $z_{j}=x_{j^{\prime}}$ or $z_{j}=y_{j^{\prime}-k}$, then $v_{j}^{\prime}=v_{j^{\prime}}$.

Match and term match $_{b}^{R_{r}(\boldsymbol{z})}$ are used to confirm that given an assignment $v$ for variables $x_{1}, \ldots, x_{k}, y_{1}, \ldots, y_{k^{\prime}}$, a tuple $\boldsymbol{z} \in Z^{a_{r}}$, and a tuple $\left(v^{\prime}{ }_{1}, \ldots, v^{\prime}{ }_{a_{r+1}}\right) \in\{\top, \perp\}^{a_{r+1}}$, that $\left(v\left(z_{1}\right), \ldots, v\left(z_{a_{r}}\right)\right)=\left(v^{\prime}{ }_{1}, \ldots, v^{\prime}{ }_{a_{r}}\right)$, since this is a crucial condition to assert that $\left[R_{r}(\boldsymbol{z})\right]^{v a_{r+1}}$ must be true (i.e. $R_{r}(\boldsymbol{z})$ is true iff $v_{a_{r+1}}=\top$ ).

$T^{!}\left(\operatorname{match}_{b}^{R_{r}(z)}\right)$ is defined in the following way:

$T^{!}\left(\right.$match $\left._{0}^{R_{r}(\boldsymbol{z})}\right)=c \cdot !$ addhyph $\cdot$ ! ather $_{r}$ and for $b>0$ and $z_{b}=y_{l-k}$, $T^{!}\left(\operatorname{match}_{b}^{R_{r}(\boldsymbol{z})}\right)=$

$c . \cdot\left[c \cdot !\right.$ mphypoth $\left.\cdot T^{!}\left(\operatorname{match}_{b-1}^{R_{r}(\boldsymbol{z})}\right)\right] \cdot !\left[\operatorname{tran} \cdot\left[\operatorname{tran} \cdot \operatorname{project}_{l}^{\rho_{1}} \cdot \operatorname{match}\left(y_{l}, b\right)\right] \cdot \operatorname{replace}_{b}^{a_{r}+1}\right]$

We can see by induction on $b$ that for every $0 \leq b \leq a_{r}$,

Match, !gather $:_{r}$ gather $_{r}:_{1}\left(\left[p_{1}\right]^{v_{1}^{\prime}} \wedge \cdots \wedge\left[p_{a_{r}}\right]^{v_{a_{r}}^{\prime}} \wedge\left[R_{r}\right]^{v_{a_{r}+1}}\right) \vdash$

$$
\begin{aligned}
& \vdash T^{!}\left(\operatorname{match}_{b}^{R_{r}(\boldsymbol{z})}\right):_{2} \operatorname{match}_{b}^{R_{r}(\boldsymbol{z})}:_{1}\left(\bigwedge\left[x_{i}\right]^{v_{i}} \wedge \bigwedge\left[y_{i}\right]^{v_{k+i}} \rightarrow\right. \\
& \left.\quad \rightarrow\left(o k_{1} \wedge \cdots \wedge o k_{b} \wedge\left[p_{b+1}\right]^{v_{b+1}^{\prime}} \wedge \cdots \wedge\left[p_{a_{r}}\right]^{v_{a_{r}}^{\prime}} \wedge\left[R_{r}\right]^{v_{a_{r}+1}}\right)\right)
\end{aligned}
$$

if and only if

$$
\begin{aligned}
\text { Match, } \text { gather }_{r}:_{1} & \left(\left[p_{1}\right]^{v_{1}^{\prime}} \wedge \cdots \wedge\left[p_{a_{r}}\right]^{v_{a_{r}}^{\prime}} \wedge\left[R_{r}\right]^{v_{a_{r}+1}}\right) \vdash \\
& \vdash \operatorname{match}_{b}^{R_{r}\left(z_{1}, \ldots, z_{a_{r}}\right)}:_{1}\left(\bigwedge\left[x_{i}\right]^{v_{i}} \wedge \wedge\left[y_{i}\right]^{v_{k+i}} \rightarrow\right. \\
& \left.\rightarrow\left(\text { ok }_{1} \wedge \cdots \wedge o k_{b} \wedge\left[p_{b+1}\right]^{v_{b+1}^{\prime}} \wedge \cdots \wedge\left[p_{a_{r}}\right]^{v_{a_{r}}^{\prime}} \wedge\left[R_{r}\right]^{v_{a_{r}+1}}\right)\right),
\end{aligned}
$$

which in turn, as we have seen above, is true if and only if for every $j \in\left[a_{r}\right]$ and $j^{\prime} \in\left[k+k^{\prime}\right]$, if $z_{j}=x_{j^{\prime}}$ or $z_{j}=y_{j^{\prime}-k}$, then $v_{j}^{\prime}=v_{j^{\prime}}$. 
Using the terms (and formulas) we have defined above, we can construct terms $T^{a}$, where $0<a \leq \rho_{1}$ and eventually $t^{\phi}$ :

Let $\Psi=\left\{\psi_{1}, \ldots, \psi_{l}\right\}$ be an ordering of all subformulas of $\psi$ and of variables $x_{1}, \ldots, x_{k}, y_{1}, \ldots, y_{k^{\prime}}$, which extends the ordering $x_{1}, \ldots, x_{k}, y_{1}, \ldots, y_{k^{\prime}}$, such that if $a<b$, then $\left|\psi_{a}\right| \leq\left|\psi_{b}\right| .^{8}$ Furthermore, $\rho_{0}=\left|\left\{a \in[l]|| \psi_{a} \mid=0\right\}\right|$ $\left(=k+k^{\prime}\right)$ and $\rho_{1}=\left|\left\{a \in[l]|| \psi_{a} \mid=1\right\}\right|$.

Let $T^{1}=$ value $_{z_{1}}$ and for every $1<a \leq \rho_{0}, T^{a}=\left[\right.$ append $\cdot T^{a-1} \cdot$ value $\left._{z_{a}}\right]$. It is not hard to see that for $v_{1}, \ldots, v_{k} \in\{\top, \perp\}$,

$$
\text { value }_{z_{1}}:_{1}\left[z_{1}\right]^{v_{1}}, \ldots, \text { value }_{z_{k}}:_{1}\left[z_{k}\right]^{v_{k}} \vdash T^{\rho_{0}}:_{1}\left(\left[z_{1}\right]^{v_{1}} \wedge \cdots \wedge\left[z_{k}\right]^{v_{k}}\right) .
$$

For every $a \in[l]$,

if $\psi_{a}=R_{r}\left(z_{1}^{a}, \ldots, z_{a_{r}}^{a}\right)$, then

$$
\begin{gathered}
\text { Eval }_{a}=\operatorname{truth}_{a}:_{2}\left(\left[\text { match }_{a_{r}}^{\psi_{a}} \cdot T^{\rho_{0}}\right]:_{1}\left(o k_{1} \wedge \cdots \wedge o k_{a_{r}} \wedge\left[R_{r}\right]^{\top}\right) \rightarrow\left[\psi_{a}\right]^{\top}\right) \wedge \\
\wedge \text { truth }_{a}:_{2}\left(\left[\text { match }_{a_{r}}^{\psi_{a}} \cdot T^{\rho_{0}}\right]:_{1}\left(o k_{1} \wedge \cdots \wedge o k_{a_{r}} \wedge\left[R_{r}\right]^{\perp}\right) \rightarrow\left[\psi_{a}\right]^{\perp}\right)
\end{gathered}
$$

if $\psi_{a}=\neg \gamma$, then

$$
\text { Eval }_{a}=\operatorname{truth}_{a}:_{2}\left([\gamma]^{\top} \rightarrow\left[\psi_{a}\right]^{\perp}\right) \wedge \operatorname{truth}_{a}:_{2}\left([\gamma]^{\perp} \rightarrow\left[\psi_{a}\right]^{\top}\right) ;
$$

if $\psi_{a}=\gamma \vee \delta$, then

$$
\begin{gathered}
\text { Eval }_{a}=\text { truth }_{a}:_{2}\left([\gamma]^{\top} \wedge[\delta]^{\top} \rightarrow\left[\psi_{a}\right]^{\top}\right) \wedge \text { truth }_{a}:_{2}\left([\gamma]^{\top} \wedge[\delta]^{\perp} \rightarrow\left[\psi_{a}\right]^{\top}\right) \\
\wedge \text { truth }_{a}:_{2}\left([\gamma]^{\perp} \wedge[\delta]^{\top} \rightarrow\left[\psi_{a}\right]^{\top}\right) \wedge \text { truth }_{a}:_{2}\left([\gamma]^{\perp} \wedge[\delta]^{\perp} \rightarrow\left[\psi_{a}\right]^{\perp}\right)
\end{gathered}
$$

if $\psi_{a}=\gamma \wedge \delta$, then

$$
\begin{gathered}
\text { Eval }_{a}=\text { truth }_{a}:_{2}\left([\gamma]^{\top} \wedge[\delta]^{\top} \rightarrow\left[\psi_{a}\right]^{\top}\right) \wedge \text { truth }_{a}:_{2}\left([\gamma]^{\top} \wedge[\delta]^{\perp} \rightarrow\left[\psi_{a}\right]^{\perp}\right) \\
\wedge \text { truth }_{a}:_{2}\left([\gamma]^{\perp} \wedge[\delta]^{\top} \rightarrow\left[\psi_{a}\right]^{\perp}\right) \wedge \text { truth }_{a}:_{2}\left([\gamma]^{\perp} \wedge[\delta]^{\perp} \rightarrow\left[\psi_{a}\right]^{\perp}\right)
\end{gathered}
$$

if $\psi_{a}=\gamma \rightarrow \delta$, then

$$
\begin{gathered}
\text { Eval }_{a}=\text { truth }_{a}:_{2}\left([\gamma]^{\top} \wedge[\delta]^{\top} \rightarrow\left[\psi_{a}\right]^{\top}\right) \wedge \text { truth }_{a}:_{2}\left([\gamma]^{\top} \wedge[\delta]^{\perp} \rightarrow\left[\psi_{a}\right]^{\perp}\right) \\
\wedge \text { truth }_{a}:_{2}\left([\gamma]^{\perp} \wedge[\delta]^{\top} \rightarrow\left[\psi_{a}\right]^{\top}\right) \wedge \text { truth }_{a}:_{1}\left([\gamma]^{\perp} \wedge[\delta]^{\perp} \rightarrow\left[\psi_{a}\right]^{\top}\right) .
\end{gathered}
$$

Let Eval $=\bigwedge_{a=\rho_{0}+1}^{l} E^{\prime} v a l_{a}$.

For $\rho_{0}<a \leq \rho_{1}$, we define gathrel $_{a}$ in the following way:

$$
\text { gathrel }_{\rho_{0}+1}=c \cdot \cdot T^{!}\left(\text {match }_{a_{r_{a}}}^{\psi_{a}}\right)
$$

and for $\rho_{0}+1<a \leq \rho_{1}$,

$$
\text { gathrel }_{\rho_{0}+1}=\text { appendconc }_{\text {gathrel }} \text { at-1 }_{a} \cdot\left[c \cdot T^{!}\left(\text {match }_{a_{a_{a}}}^{\psi_{a}}\right)\right] .
$$

\footnotetext{
${ }^{8}$ assume a $|\cdot|$, such that $\left|x_{j}\right|=\left|y_{j}\right|=0,\left|R_{j}\left(v_{1}, \ldots, v_{a_{j}}\right)\right|=1$ and if $\gamma$ is a proper subformula of $\delta$, then $|\gamma|<|\delta|$
} 
Then,

$$
T^{\rho_{0}+1}=\text { replace }_{1}^{\rho_{1}-\rho_{0}} \cdot \text { truth }_{\rho_{0}+1} \cdot\left[\text { gathrel }_{\rho_{1}} ! T^{\rho_{0}}\right]
$$

and for $\rho_{0}+1<a \leq \rho_{1}$,

$$
T^{a}=\operatorname{replace}_{a}^{\rho_{1}-\rho_{0}} \cdot \operatorname{truth}_{\rho_{0}+1} \cdot T^{a-1} .
$$

if $\psi_{a}=\neg \psi_{2}$, then

$$
T^{a}=\text { hypappend } \cdot\left[\text { trans } \cdot \operatorname{proj}_{j}^{a-\rho_{0}-1} \cdot \text { truth }_{a}\right] \cdot T^{a-1} \text { and }
$$

if $\psi_{a}=\psi_{b} \circ \psi_{c}$, then

$$
T^{a}=\text { hypappend } \cdot\left[\text { trans } \cdot\left[\text { appendconc } \cdot \operatorname{proj}_{b}^{a-\rho_{0}-1} \cdot \operatorname{proj}_{c}^{a-1}\right] \cdot \text { truth }_{a}\right] \cdot T^{a-1} .
$$

We then define $t^{\phi}=\left[\right.$ right $\left.\cdot T^{l}\right]$.

Lemma 5. For every $b \in\left[\rho_{1}\right], j \in\left[a_{r_{b}}\right]$, let $\boldsymbol{l}^{\boldsymbol{b}}=\left(l^{b}{ }_{1}, \ldots, l^{b}{ }_{a_{r_{b}}}\right) \in\left\{p_{j}, \neg p_{j}\right\}^{a_{r_{b}}}$ and $v^{b} \in\{\top, \perp\}$. Assume that for every $b_{1}, b_{2} \in\left[\rho_{1}\right]$, if $r_{b_{1}}=r_{b_{2}}$ and $\boldsymbol{l}^{\boldsymbol{b}_{\mathbf{1}}}=\boldsymbol{l}^{\boldsymbol{b}_{\mathbf{2}}}$, then it must also be the case that $v^{b_{1}}=v^{b_{2}}$. Then, ${ }^{9}$

$$
\bigwedge_{b \in\left[\rho_{1}\right]} ! \text { gather }_{r_{b}}:_{2} \text { gather }_{r_{b}}:_{1}\left(\boldsymbol{l}^{b} \wedge\left[R_{r_{b}}\right]^{b}\right) \wedge M a t c h \wedge E v a l \wedge \bigwedge_{z \in Z} \text { val }_{z}:_{1}[z]^{v_{z}} \vdash t^{\phi}:_{2}[\phi]^{\top}
$$

if and only if $\mathcal{M} \models \phi$ for every model $\mathcal{M}$ with universe $\{\top, \perp\}$ and interpretation $\mathcal{I}$ such that

- for every $z \in Z, v_{z}=\mathcal{I}(z)$,

- for every $b \in\left[\rho_{1}\right], \mathcal{M} \models R_{r_{b}}\left(f\left(l_{1}^{b}\right), \ldots, f\left(l_{a_{r_{b}}}^{b}\right)\right)$ iff $v^{b}=\top$,

where for all $j \in \alpha, f\left(p_{j}\right)=\top$ and $f\left(\neg p_{j}\right)=\perp$.

Proof. The if direction is not hard to see by (induction on) the construction of the terms $T^{a}, t^{\phi}$. For the other direction, notice that a $*$-calculus derivation for

$$
\begin{aligned}
& \bigwedge_{b \in\left[\rho_{1}\right]} \text { lgather }_{r_{b}:_{2}} \text { gather }_{r_{b}: 1}\left(\boldsymbol{l}^{\boldsymbol{b}} \wedge\left[R_{r_{b}}\right]^{v^{b}}\right) \\
& \text { Match, Eval, } \bigwedge_{z \in Z} \operatorname{val}_{z}:_{1}[z]^{v_{z}} \vdash t^{\phi}:_{2}[\phi]^{\top}
\end{aligned}
$$

gives on the right hand side a derivation of

$$
\bigwedge_{b \in\left[\rho_{1}\right]} \text { gather }_{r_{b}: 1}\left(\boldsymbol{l}^{\boldsymbol{b}} \wedge\left[R_{r_{b}}\right]^{v^{b}}\right), \text { Match, Eval } \#_{2}, \bigwedge_{z \in Z} \text { val }_{z}:_{1}[z]^{v_{z}} \vdash[\phi]^{\top}
$$

Some $\chi=\left[R_{r}\left(\boldsymbol{z}_{r}^{a}\right)\right]^{\triangle}$, where $R_{r}\left(\boldsymbol{z}_{r}^{a}\right)=\psi_{a}$, a subformula of $\phi$, can be derived from the assumptions above only if $\left[\right.$ match $\left._{a_{r_{a}}}^{\psi_{a}} \cdot T^{\rho_{0}}\right]:_{1}\left(\boldsymbol{o k} \wedge\left[R_{r_{a}}\right]^{\triangle}\right)$ can be

\footnotetext{
${ }^{9}$ For convenience and to keep the notation tidy, we identify $\boldsymbol{l}^{b}$ with $l_{1}^{b} \wedge \cdots \wedge l_{a_{r_{b}}}^{b}$ and ok with $o k_{1} \wedge \cdots \wedge o k_{a_{r_{b}}}$.
} 
derived as well - notice that the assumptions cannot be inconsistent and we can easily adjust a model that does not satisfy $\left[\operatorname{match}_{a_{r_{a}}}^{\psi_{a}} \cdot T^{\rho_{0}}\right]:_{1}\left(\boldsymbol{o k} \wedge\left[R_{r_{a}}\right]^{\triangle}\right)$ so that it does not satisfy $\chi$ either, by simply changing the truth value of $\chi$.

The derivation of match $_{a_{r_{a}}}^{\psi_{a}}:_{1}\left(\boldsymbol{o k} \wedge\left[R_{r_{a}}\right]^{\triangle}\right)$ is not affected by Eval ${ }^{\#_{2}}$ : if there is a model that satisfies all assumptions except for Eval $\#_{2}$ and not match $_{a_{r_{a}}:_{1}}^{\psi_{a}}\left(\boldsymbol{o k} \wedge\left[R_{r_{a}}\right]^{\triangle}\right)$, we can assume the strong evidence property and change the truth-values of every $\left[\psi_{b}\right]^{\triangle^{\prime}}$ to true, so the new model satisfies all the assumptions and not $\left[\right.$ match $\left._{a_{r_{a}}}^{\psi_{a}} \cdot T^{\rho_{0}}\right]:_{1}\left(\boldsymbol{o k} \wedge\left[R_{r_{a}}\right]^{\triangle}\right)$.

Therefore we have a $*$-calculus derivation of $\left[\operatorname{match}_{a_{r_{a}}}^{\psi_{a}} \cdot T^{\rho_{0}}\right]::_{1}\left(\boldsymbol{o k} \wedge\left[R_{r_{a}}\right]^{\triangle}\right)$ and since gather $_{r}$ only appears once in match $_{a_{r_{a}}}^{\psi_{a}}$, there is some $b \in\left[\rho_{1}\right]$ such that (see Lemma 2)

${\text { gather } r_{b}:_{1}}\left(\boldsymbol{l}^{\boldsymbol{b}} \wedge\left[R_{r_{b}}\right]^{b^{b}}\right)$, Match $, \bigwedge_{z \in Z} \operatorname{val}_{z}:_{1}[z]^{v_{z}} \vdash\left[\right.$ match $\left._{a_{r_{a}}}^{\psi_{a}} \cdot T^{\rho_{0}}\right]:_{1}\left(\boldsymbol{o k} \wedge\left[R_{r_{a}}\right]^{\triangle}\right)$

Similarly, we can remove the terms from this derivation, so

$$
\boldsymbol{l}^{\boldsymbol{b}},\left[R_{r_{b}}\right]^{v^{b}}, \text { Match }^{\# 1}, \bigwedge_{z \in Z}[z]^{v_{z}} \vdash \boldsymbol{o k} \wedge\left[R_{r_{a}}\right]^{\triangle}
$$

From which it is not hard to see that for all $z \in Z, v^{b}=\triangle$, so every firstorder model as described in the Lemma satisfies $\chi$. Then it is not hard to see by induction that all such models satisfy all $\left[\psi_{a}\right]^{\triangle}$ derivable from these same assumptions.

Now to construct the actual formula the reduction gives. For this let $\rho$ be a fixed justification variable. We define the following formulas.

$$
\begin{aligned}
& \text { start }=\neg[\text { active }] \wedge \rho:_{3}\left([\text { active }] \wedge \bigwedge_{a \in[\alpha]} \operatorname{var}_{a}:_{1} \neg p_{a}\right) \\
& \text { forward }_{A}=\rho:_{4}\left(\bigvee_{a \in[\alpha]} \operatorname{var}_{a}:_{1} \neg p_{a} \wedge[\text { active }] \rightarrow \rho:_{3}[\text { active }]\right) \\
& \text { forward }_{B}=\rho:_{4} \bigwedge_{a \in[\alpha]}\left(\bigwedge_{b \in[a-1]} \operatorname{var}_{b}:_{1} p_{b} \wedge \operatorname{var}_{a}:_{1} \neg p_{a} \wedge[\text { active }]\right. \\
&\left.\rightarrow \rho:_{3}\left(\bigwedge_{b \in[a-1]} \operatorname{var}_{b}:_{1} \neg p_{b} \wedge \operatorname{var}_{a}:_{1} p_{a}\right)\right) \\
& \text { forward }_{C}=\rho:_{4} \bigwedge_{a \in[\alpha]}\left(\bigvee_{b \in[a-1]} \operatorname{var}_{b}:_{1} \neg p_{b} \wedge \operatorname{var}_{a}:_{1} \neg p_{a} \wedge[\text { active }]\right. \\
&\left.\rightarrow \rho:_{3} \operatorname{var}_{a}:_{1} \neg p_{a}\right)
\end{aligned}
$$




$$
\begin{aligned}
& \operatorname{forward}_{D}=\rho:_{4} \bigwedge_{a \in[\alpha]}\left(\bigvee_{b \in[a-1]} \operatorname{var}_{b}:_{1} \neg p_{b} \wedge \operatorname{var}_{a}:_{1} p_{a} \wedge[\text { active }]\right. \\
& \left.\rightarrow \rho:_{3} \operatorname{var}_{a}: 1 p_{a}\right) \\
& \text { end }=\rho:_{4}\left(\bigwedge_{a \in \alpha} \text { var }:_{1} p_{a} \wedge[\text { active }] \rightarrow \rho:_{4} \neg[\text { active }]\right) \\
& \text { choice }_{R}=\rho:_{4}\left([\text { active }] \rightarrow \text { rel }_{r}:_{1}\left[R_{r}\right]^{\top} \vee \text { rel }_{r}:_{1}\left[R_{r}\right]^{\perp}\right) \\
& \text { choice }_{V}=\rho:_{4}\left(\neg[\text { active }] \rightarrow \bigwedge_{z \in X}\left(\text { value }_{z}:_{1}[z]^{\top} \vee \text { value }_{z}:_{1}[z]^{\perp}\right)\right. \\
& \left.\wedge \bigwedge_{z \in Y}\left(\text { value }_{z}:_{1}[z]^{\top} \wedge \text { value }_{z}:_{1}[z]^{\perp}\right)\right) \\
& \text { test }=\rho:_{4}\left(\neg[\text { active }] \rightarrow \text { Match } \wedge \text { Eval } \wedge \neg t^{\phi}:_{2}[\neg \phi]^{T}\right)
\end{aligned}
$$

Then, $\phi_{F O}^{J}$, the formula constructed by the reduction is the conjunction of these formulas above:

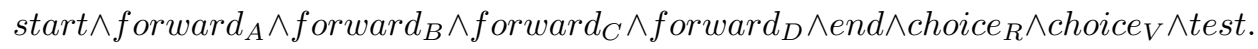

Theorem 3. $\phi_{F O}^{J}$ is J-satisfiable if and only if $\phi$ is satisfiable by a two-element first-order model.

Proof. First, assume $\phi$ is satisfiable by two-element first-order model, say $\mathcal{M}$ with interpretation $\mathcal{I}$, and assume that for every $a \in[k], \mathcal{I}\left(x_{a}\right)$ is such that $\mathcal{M} \models \forall y_{1}, \ldots, \forall y_{k^{\prime}} \psi$. We construct a $J$-model for $\phi_{F O}^{J}$ :

$$
\mathcal{M}_{J}=\left(W, R_{1}, R_{2}, R_{3}, R_{4}, \mathcal{E}, \mathcal{V}\right) \text {, where: }
$$

- $W=\left\{\sigma \in \mathbb{N} \mid \sigma+2 \in\left[2^{\alpha}+2\right]\right\}$ (i.e. $\sigma \in\left\{-1,0,1,2, \ldots 2^{\alpha}\right\}$ );

- $R_{1}=R_{2}=\emptyset, R_{3}=\left\{(\sigma, \sigma+1) \mid \sigma<2^{\alpha}\right\} \cup\left\{\left(2^{\alpha}, 2^{\alpha}\right)\right\}$, and

$R_{4}=\left\{\left(\sigma, \sigma^{\prime}\right) \mid \sigma<\sigma^{\prime}\right\} \cup\left\{\left(2^{\alpha}, 2^{\alpha}\right)\right\}$;

$-\mathcal{E}$ is minimal such that

- $\mathcal{E}_{3}(\rho, \chi)=\mathcal{E}_{4}(\rho, \chi)=W$ for any formula $\chi$,

- $\mathcal{E}_{1}\left(\operatorname{var}_{a}, p_{a}\right)=\left\{\sigma \in W \mid \sigma+1 \in\left[2^{\alpha}\right]\right.$ and $\left.\operatorname{bin}_{a}(\sigma)=1\right\}$

- $\mathcal{E}_{1}\left(\operatorname{var}_{a}, \neg p_{a}\right)=\left\{\sigma \in W \mid \sigma+1 \in\left[2^{\alpha}\right]\right.$ and $\left.\operatorname{bin}_{a}(\sigma)=0\right\}$,

- $\mathcal{E}_{1}\left(\right.$ rel $\left._{r},\left[R_{r}\right]^{\top}\right)=\left\{\sigma \in W \mid \sigma+1 \in\left[2^{\alpha}\right]\right.$ and $\left.\mathcal{M} \models R_{r}\left(b_{0 i n}(\sigma), \ldots, b_{i n} a_{a_{r}}(\sigma)\right)\right\}$,

- $\mathcal{E}_{1}\left(\right.$ rel $\left._{r},\left[R_{r}\right]^{\perp}\right)=\left\{\sigma \in W \mid \sigma+1 \in\left[2^{\alpha}\right]\right.$ and $\left.\mathcal{M} \not \models R_{r}\left(\operatorname{bin}_{0}(\sigma), \ldots, \operatorname{bin}_{a_{r}}(\sigma)\right)\right\}$,

- for every $a \in[k], \mathcal{E}_{1}\left(\right.$ value $\left._{x_{a}},\left[x_{a}\right]^{\top}\right)=\left\{2^{\alpha}\right\}$, if $\mathcal{I}\left(x_{a}\right)=\top$ and $\emptyset$ otherwise,

- for every $a \in[k], \mathcal{E}_{1}\left(\right.$ value $\left._{x_{a}},\left[x_{a}\right]^{\perp}\right)=\left\{2^{\alpha}\right\}$, if $\mathcal{I}\left(x_{a}\right)=\perp$ and $\emptyset$ otherwise,

- for every $a \in\left[k^{\prime}\right], \mathcal{E}_{1}\left(\right.$ value $\left._{y_{a}},\left[y_{a}\right]^{\top}\right)=\mathcal{E}_{i}\left(\right.$ value $\left._{y_{a}},\left[y_{a}\right]^{\perp}\right)=\left\{2^{\alpha}\right\}$, and 
- $\mathcal{M}_{J}, 2^{\alpha} \models$ Match, Eval;

$-\mathcal{V}([$ active $])=\left\{\sigma \in W \mid \sigma+1 \in\left[2^{\alpha}\right]\right\}$ and for any other propositional variable $q, V(q)=\emptyset$.

It is not hard to verify that $\mathcal{M}_{J},-1 \models \phi_{F O}^{J}$, as long as we establish that $\mathcal{M}_{J}, 2^{\alpha} \not \forall t^{\phi}:_{2}[\neg \phi]^{T}$, for which it is enough that $2^{\alpha} \notin \mathcal{E}_{j}\left(t^{\phi},[\neg \phi]^{\top}\right)$. $S=$

The definition of $\mathcal{E}$ is equivalent to $\sigma \in \mathcal{E}_{g}(s, \chi) \Leftrightarrow S \vdash_{*} \sigma *_{g}(s, \chi)$, where

$$
\begin{gathered}
\left\{w *_{3}(\rho, F) \mid w \in W, F \text { a formula }\right\} \cup\left\{w *_{4}(\rho, F) \mid w \in W, F \text { a formula }\right\} \cup \\
\left\{w *_{1}\left(\text { var }_{a}, p_{a}\right) \mid w+1 \in\left[2^{\alpha}\right] \text { and } \operatorname{bin}_{a}(w)=1\right\} \cup \\
\left\{w *_{1}\left(\text { var }_{a}, \neg p_{a}\right) \mid w+1 \in\left[2^{\alpha}\right] \text { and } \operatorname{bin}_{a}(w)=0\right\} \cup \\
\left\{w *_{1}\left(\text { rel }_{r},\left[R_{r}\right]^{\top}\right) \mid w+1 \in\left[2^{\alpha}\right] \text { and } \mathcal{M} \models R_{r}\left(\operatorname{bin}_{0}(w), \ldots, \operatorname{bin}_{a_{r}}(w)\right)\right\} \cup \\
\left\{w *_{1}\left(\text { rel }_{r},\left[R_{r}\right]^{\perp}\right) \mid w+1 \in\left[2^{\alpha}\right] \text { and } \mathcal{M} \not R_{r}\left(\text { bin }_{0}(w), \ldots, \text { bin }_{a_{r}}(w)\right)\right\} \cup \\
\left\{2^{\alpha} *_{1}\left(\text { value }_{x_{a}},\left[x_{a}\right]^{\top}\right) \mid a \in[k], \mathcal{I}\left(x_{a}\right)=\top\right\} \cup \\
\left\{2^{\alpha} *_{1}\left(\text { value }_{x_{a}},\left[x_{a}\right]^{\perp}\right) \mid a \in[k], \mathcal{I}\left(x_{a}\right)=\perp\right\} \cup \\
\left\{2^{\alpha} *_{1}\left(\text { value }_{y_{a}},\left[y_{a}\right]^{\top}\right) \mid a \in\left[k^{\prime}\right]\right\} \cup\left\{2^{\alpha} *_{1}\left(\text { value }_{y_{a}},\left[y_{a}\right]^{\perp}\right) \mid a \in\left[k^{\prime}\right]\right\} \cup \\
\left\{2^{\alpha} e \mid e \in * E v a l \cup * \text { Match }\right\}
\end{gathered}
$$

Then, $2^{\alpha} \in \mathcal{E}_{2}\left(t^{\phi},[\neg \phi]^{\top}\right)$ iff $S \vdash_{*} 2^{\alpha} *_{2}\left(t^{\phi},[\neg \phi]^{\top}\right)$. Notice the following: since $t^{\phi}$ does not have $\rho$ as a subterm, the $*$-expressions in

$$
\left\{w *_{3}(\rho, F) \mid w \in W, F \text { a formula }\right\} \cup\left\{w *_{4}(\rho, F) \mid w \in W, F \text { a formula }\right\}
$$

cannot be a part of a derivation for $S \vdash_{*} 2^{\alpha} *_{2}\left(t^{\phi},[\neg \phi]^{\top}\right)$.

Since $1 \hookleftarrow 2 \hookleftarrow 3$ and 1,2 do not interact with any agents in any other way, for any term $s$ with no !, if for some $a$ or $r, v a r_{a}$ or $r e l_{r}$ are subterms os $s$, if $S \vdash_{*} w s:_{a} \chi$, then $a=1,0 \leq w<2^{\alpha}$, and $\{w e \in S\} \vdash_{*} w s:_{1} \chi \cdot t^{\phi}$ includes exactly one ! gather $r_{r_{b}}$ for every $b$ and one of value $_{z}$ for every $z \in Z$. Therefore, if $S \vdash_{*} 2^{\alpha} *_{2}\left(t^{\phi},[\neg \phi]^{\top}\right)$, then there are

$$
\bigwedge_{b \in\left[\rho_{1}\right]} ! \text { gather }_{r_{b}}:_{2} \text { gather }_{r_{b}}:_{1} \Phi \wedge \text { Match } \wedge \text { Eval } \wedge \bigwedge_{z \in Z} \text { val }_{z}:_{1}[z]^{v_{z}} \vdash t^{\phi}:_{2}[\neg \phi]^{\top}
$$

and by Lemma $5, \mathcal{M} \models \neg \phi$, a contradiction.

On the other hand, let there be some $\mathcal{M}_{J}^{\prime}$ where $\phi^{J}$ is satisfied. Then, we name -1 a state where $\mathcal{M}_{J}^{\prime},-1 \models \phi^{J}$ and let $-1 R_{3} 0 R_{3} 1 R_{3} \cdots R_{3} 2^{\alpha}$. Then,

$-\mathcal{E}_{1}\left(\operatorname{var}_{a}, p_{a}\right) \subseteq\left\{\sigma \in W \mid \sigma+1 \in\left[2^{\alpha}\right]\right.$ and $\left.\operatorname{bin}_{a}(\sigma)=1\right\}$,

$-\mathcal{E}_{1}\left(\operatorname{var}_{a}, \neg p_{a}\right) \subseteq\left\{\sigma \in W \mid \sigma+1 \in\left[2^{\alpha}\right]\right.$ and $\left.\operatorname{bin}_{a}(\sigma)=0\right\}$,

- $\mathcal{M}_{J}, 2^{\alpha} \models$ Match, Eval and for every $a \in\left[k^{\prime}\right]$,

$\mathcal{M}_{J}, 2^{\alpha} \models$ value $_{y_{a}}:_{1}\left[y_{a}\right]^{\top}$, alue $_{y_{a}}:_{1}\left[y_{a}\right]^{\perp}$; 
as we can see by induction on $\sigma$ - the conditions on $A_{1}\left(v_{a r}, p_{a}\right), A_{1}\left(v a r_{a}, \neg p_{a}\right)$ as imposed by forward $_{B}$, forward forward $_{D}$ are positive. Notice here that if for some $0 \leq w<2^{\alpha}-1, w \in \bigcap_{a \in \alpha} \mathcal{E}_{1}\left(\operatorname{var}_{a}, p_{a}\right)$, then we have a contradiction: $w+1 \models \neg[$ active $]$ and if $w$ is minimal for this to happen, then $w \models[$ active], so since there is some $a$ s.t. $w \in \mathcal{E}_{1}\left(\right.$ var $\left._{a}, \neg p_{a}\right), w+1 \models[$ active $]$ (by forward for $_{A}$ ).

Then, $\left\{w \mid w+1 \in\left[2^{\alpha}\right]\right\} \subseteq \mathcal{E}_{1}\left(\right.$ rel $\left._{r},\left[R_{r}\right]^{\top}\right) \cup \mathcal{E}_{1}\left(\right.$ rel $\left._{r},\left[R_{r}\right]^{\perp}\right)$ and then we can define a first-order model $\mathcal{M}$ such that:

$-\mathcal{E}_{1}\left(\operatorname{rel}_{r},\left[R_{r}\right]^{\top}\right) \subseteq\left\{\sigma \in W \mid \sigma+1 \in\left[2^{\alpha}\right]\right.$ and $\left.\mathcal{M} \models R_{r}\left(\operatorname{bin}_{0}(\sigma), \ldots, \operatorname{bin}_{a_{r}}(\sigma)\right)\right\}$,

$-\mathcal{E}_{1}\left(r e l_{r},\left[R_{r}\right]^{\perp}\right) \subseteq\left\{\sigma \in W \mid \sigma+1 \in\left[2^{\alpha}\right]\right.$ and $\left.\mathcal{M} \forall \forall R_{r}\left(\operatorname{bin}_{0}(\sigma), \ldots, \operatorname{bin}_{a_{r}}(\sigma)\right)\right\}$,

- for every $a \in[k], \mathcal{E}_{1}$ value $\left._{x_{a}},\left[x_{a}\right]^{\top}\right) \subseteq\left\{2^{\alpha}\right\}$, if $\mathcal{I}\left(x_{a}\right)=\top$ and $\emptyset$ otherwise,

- for every $a \in[k], \mathcal{E}_{1}\left(\right.$ value $\left._{x_{a}},\left[x_{a}\right]^{\perp}\right) \subseteq\left\{2^{\alpha}\right\}$, if $\mathcal{I}\left(x_{a}\right)=\perp$ and $\emptyset$ otherwise,

Since it must be the case that $\mathcal{M}_{J}, 2^{\alpha} \not \forall t^{\phi}:_{2}[\neg \phi]$, it cannot be the case that

$$
\bigwedge_{b \in\left[\rho_{1}\right]} ! \text { gather }_{r_{b}}:_{2} \text { gather }_{r_{b}}:_{1} \Phi \wedge \text { Match } \wedge \text { Eval } \wedge \bigwedge_{z \in Z} \text { val }_{z}:_{1}[z]^{v_{z}} \vdash t^{\phi}:_{2}[\neg \phi]^{\top}
$$

and since $\mathcal{M}$ satisfies the conditions from Lemma $5, \mathcal{M} \not \models \neg \phi$.

Theorem 2 is then a direct consequence.

\section{Final Remarks}

We gave two lower bounds for the complexity of the satisfiability problem for Justification Logic. Theorem 1 gives a general lower bound which applies to all logics in the family, while Theorem 2 gives a lower bound for a specific logic in the family. From a technical point of view, the reduction from a fragment of QBF that we used for the first result is a simplification of the reduction from a fragment of First-order Satisfiability that we used for the second result.

The merits of the general $\Sigma_{2}^{p}$-hardness result is that we established an (expected) lower bound for all the logics in the family, which uses fewer assumptions than a previous proof of the same bound (for single-agent logics) by Buss and Kuznets in [8]. That is, we require a schematic and axiomatically appropriate constant specification, while the proof in [8] requires that it is also schematically injective: each constant justifies at most one scheme. It is perhaps a subtle distinction, but it means that for the first time we established this lower bound for justification logics J, JT, JD, JD4, and LP, the versions of these single-agent logics with the total constant specification (i.e. the one where all constants justify all axioms). ${ }^{10}$ The necessity of these properties of the constant specification for these results and their full effects on the complexity of Justification Logic remain to be seen, but some insightful observations were made in [8].

The NEXP-hardness result we presented in this paper makes the general NEXP-upper bound from [4] tight, thus answering the open question from there about whether there exists a NEXP-complete logic or the upper bound can be

\footnotetext{
${ }^{10}$ If nothing else, this should simplify some of the notation.
} 


$$
\frac{\sigma T \diamond_{i} \psi}{\sigma \cdot(g, i) T \psi}
$$

where $(g, i)$ is new;

$$
\frac{\sigma F \square_{i} \psi}{\sigma .(g, i) F \psi}
$$

where $(g, i)$ is new;

$$
\frac{\sigma T \square_{i} \psi}{\sigma T \square_{j} \square_{i} \psi}
$$

where $0<i<j<4$;

$$
\frac{\sigma F \diamond_{i} \psi}{\sigma \cdot(g, i) F \psi}
$$

where $(g, i)$ has already appeared and $i<4$;

$$
\frac{\sigma T \square_{i} \psi}{\sigma T \triangleright_{i} \psi}
$$

where $i \in\{3,4\}$;

$$
\begin{gathered}
\frac{\sigma F \diamond_{4} \psi}{\sigma \cdot(g, 4) F \psi} \\
\sigma \cdot(g, 4) F \diamond_{4} \psi
\end{gathered}
$$

where $(g, i)$ has already appeared and $i \in\{3,4\}$;

$$
\frac{\sigma T \square_{i} \psi}{\sigma .(g, i) T \psi}
$$

where $(g, i)$ has already appeared and $i<4$;

$$
\frac{\sigma T \square_{4} \psi}{\sigma T \square_{3} \psi}
$$

$\frac{\sigma T \square_{4} \psi}{\sigma .(g, i) T \psi}$
$\sigma .(g, i) T \square_{4} \psi$

where $(g, i)$ has already appeared and $i \in\{3,4\}$;

Table 3. Tableau rules for $M_{H}$. To test $\phi$ for $M_{H}$-satisfiability, start from a branch which only contains $(0,0) T \phi$ and keep expanding according to the rules above. A branch with $\sigma T \psi$ and $\sigma F \psi$ is propositionally closed. A (possibly infinite) branch which is not propositionally closed, but is closed under the rules is an accepting branch.

improved. It also makes $J_{H}$ the first justification logic with known complexity having a harder satisfiability problem (assuming EXP $\neq$ NEXP) than its corresponding modal logic. In fact, as Proposition 4, if $M_{H}$ is the modal logic which corresponds to $J_{H}$ (the modal logic with the same frame restrictions as $J_{H}$ ), then $M_{H}$-satisfiability is in EXP: we can simulate the tableau procedure from Table 3 using an exponential time algorithm - an alternating polynomial space one actually, where we use nondeterministic existential choices to apply the tableau rules and universal choices to select exactly one prefix $\sigma \cdot(g, i)$ from $\sigma$ to explore. While Modal Satisfiability has been studied extensively, we are not aware of anyone investigating specifically the complexity of $M_{H}$-satisfiability, so we provide a brief proof.

Proposition 4. Let $M_{H}$ be the four-modalities modal logic associated with the class of frames $\left(W, R_{1}, R_{2}, R_{3}, R_{4}\right)$ where $R_{3}, R_{4}$ are serial, $R_{3} \subseteq R_{4}$, and for $(i, j) \in\{(1,2),(2,3),(4,4)\}$, if $a R_{j} b R_{i} c$, then $a R_{i} c$. Then, $M_{H}$-satisfiability is in EXP.

Proof (Brief). We first prove that the tableau procedure from Table 3 is sound and complete. From an accepting branch for $\phi$ we can construct a model for $\phi$ : let $W$ be the set of prefixes that have appeared in the branch; let $a \in \mathcal{V}(p)$ iff $a T p$ has appeared in the branch, let for $i=1,2,3,4, r_{i}=\{(a, a .(g, i)) \in W \times W\}$, for $i=1,2, R_{i}=r_{i}, R_{3}$ is the transitive closure of $r_{3}$, and $R_{4}$ is the transitive closure of $r_{3} \cup r_{4}$. It is not hard to verify that model $\mathcal{M}=\left(W, R_{1}, R_{2}, R_{3}, R_{4}\right)$ satisfies all necessary conditions and that $\mathcal{M},(0,0) \models \phi$ - by inductively proving that if $a T \psi$ in the branch then $\mathcal{M}, a \models \psi$ and if $a F \psi$ in the branch then $\mathcal{M}, a \not \models \psi$. 
On the other hand, from a model $\mathcal{M}=\left(W, R_{1}, R_{2}, R_{3}, R_{4}\right)$ for $\phi$ we can make appropriate nondeterministic choices to construct an accepting branch for $\phi$. We map $(0,0)$ to a state $w^{(0,0)}$ such that $\mathcal{M}, w^{(0,0)} \models \phi$; then, when $\sigma .(g, i)$ appears first, it must be because of a formula of the form $\sigma T \diamond_{i} \psi$ (or $\sigma F \square_{i} \psi$, but it is essentially the same case). If $\mathcal{M}, w^{\sigma} \models \diamond_{i} \psi$, then there must be some state $w^{\sigma} R_{i} w$, such that $\mathcal{M} \models \psi$ and thus we name $w=w^{\sigma \cdot(g, i)}$. It is not hard to see that we can make such choices when applying the rules, so that if $a T \psi$ in the branch then $\mathcal{M}, w^{a} \models \psi$ if $a F \psi$ in the branch then $\mathcal{M}, w^{a} \not \forall \psi$. In fact the rules of Table 3 preserve this condition right away; we just need to make sure that the same thing happens with the propositional rules - for instance, rule $\frac{\sigma T \psi \vee \chi}{\sigma T \psi \mid \sigma \mathcal{T} \chi}$ can make an appropriate choice depending on whether $\mathcal{M}, w^{\sigma} \models \psi$ or $\mathcal{M}, w^{\sigma} \models \chi$. Thus the constructed branch cannot be propositionally closed.

What remains is to show that this tableau procedure can be simulated by an alternating algorithm which uses polynomial space - thus $M_{H}$-satisfiability is in APSPACE $=$ EXP. This can be done by applying the following method: always keep the formulas prefixed by a certain prefix $\sigma$ in memory (at first $\sigma=(0,0)$ ). First apply all the tableau rules you can on the formulas prefixed by $\sigma$ - possibly use existential nondeterministic choices for this. Then, using a universal choice, pick one of the prefixes $\sigma^{\prime}=\sigma \cdot(g, i)$ that were just constructed and replace the formulas you have in memory by the ones prefixed by $\sigma^{\prime}$. Repeat these steps until we either have $\sigma T \psi$ and $\sigma F \psi$ in memory or we see "enough" prefixes. In this case, "enough" would mean "more than $2^{6|\phi| ", ~ a s ~} \phi$ has up to $|\phi|$ subformulas, so in a branch there can only be up to $6|\phi|$ formulas prefixed by some fixed $\sigma$ - thus the algorithm only needs to use $O(|\phi|)$ memory and if it goes through $6|\phi|+1$ prefixes, then two of these have prefixed exactly the same set of formulas. If the algorithm accepts $\phi$, then we can easily reconstruct an accepting branch by just taking the union of the constructed formulas, while if there is an accepting branch, then the algorithm can explore only parts of that branch.

These results demonstrate a remarkable variability of the system. Although many logics in the family, including the single-agent justification logics, have a $\Sigma_{2}^{p}$-complete satisfiability problem, which is lower than the complexity of satisfiability for corresponding modal logics (assuming $\mathrm{PH} \neq \mathrm{PSPACE}$ ), there are logics with PSPACE-complete, EXP-complete, and as we demonstrated in this paper, NEXP-complete satisfiability problems, which in the last case is a higher complexity than the one for the corresponding modal logic (assuming EXP $\neq N E X P$ ). Still, it is important to note that even in this case the reflected fragment of the logic remains in NP and in the absence of + , in P.

Acknowledgments The author is grateful to Sergei Artemov and to an anonymous reviewer; their suggestions significantly enhanced this paper's readability.

\section{References}

1. Antonis Achilleos. A complexity question in justification logic. Journal of Computer and System Sciences, 80(6):1038 - 1045, 2014. 
2. Antonis Achilleos. Modal logics with hard diamond-free fragments. CoRR, abs/1401.5846, 2014.

3. Antonis Achilleos. On the complexity of two-agent justification logic. In Nils Bulling, Leendert van der Torre, Serena Villata, Wojtek Jamroga, and Wamberto Vasconcelos, editors, Computational Logic in Multi-Agent Systems, volume 8624 of Lecture Notes in Computer Science, pages 1-18. Springer, 2014.

4. Antonis Achilleos. Tableaux and complexity bounds for a multiagent justification logic with interacting justifications. In 12th European Conference on Multi-Agent Systems (EUMAS 2014), Lecture Notes in Artificial Intelligence, 2014.

5. Sergei Artemov. Explicit provability and constructive semantics. Bulletin of Symbolic Logic, 7(1):1-36, March 2001.

6. Sergei Artemov. Justification logic. In Steffen Hölldobler, Carsten Lutz, and Heinrich Wansing, editors, Logics in Artificial Intelligence, 11th European Conference, JELIA 2008, Dresden, Germany, September 28-October 1, 2008. Proceedings, volume 5293 of Lecture Notes in Computer Science, pages 1-4. Springer Berlin Heidelberg, 2008.

7. Sergei Artemov. The logic of justification. The Review of Symbolic Logic, 1(4):477513, December 2008.

8. Samuel R Buss and Roman Kuznets. Lower complexity bounds in justification logic. Annals of Pure and Applied Logic, 163(7):888-905, 2012.

9. Stéphane Demri. Complexity of simple dependent bimodal logics. In Roy Dyckhoff, editor, TABLEAUX, volume 1847 of Lecture Notes in Computer Science, pages 190-204. Springer, 2000.

10. Melvin Fitting. The logic of proofs, semantically. Annals of Pure and Applied Logic, 132(1):1-25, February 2005.

11. Nikolai V. Krupski. On the complexity of the reflected logic of proofs. Theoretical Computer Science, 357(1-3):136-142, July 2006.

12. Roman Kuznets. On the complexity of explicit modal logics. In Peter G. Clote and Helmut Schwichtenberg, editors, Computer Science Logic, volume 1862 of Lecture Notes in Computer Science, pages 371-383. Springer Berlin Heidelberg, 2000. Errata concerning the explicit counterparts of $\mathcal{D}$ and $\mathcal{D} 4$ are published as [15].

13. Roman Kuznets. Complexity Issues in Justification Logic. PhD thesis, CUNY Graduate Center, May 2008.

14. Roman Kuznets. Self-referentiality of justified knowledge. In Edward A. Hirsch, Alexander A. Razborov, Alexei L. Semenov, and Anatol Slissenko, editors, CSR, volume 5010 of Lecture Notes in Computer Science, pages 228-239. Springer, 2008.

15. Roman Kuznets. Complexity through tableaux in justification logic. In 2008 European Summer Meeting of the Association for Symbolic Logic, Logic Colloquium '08, Bern, Switzerland, July 3-July 8, 2008, volume 15(1) of Bulletin of Symbolic Logic, page 121. Association for Symbolic Logic, March 2009. Abstract.

16. Harry R. Lewis. Complexity results for classes of quantificational formulas. Journal of Computer and System Sciences, 21(3):317 - 353, 1980.

17. Robert [S.] Milnikel. Derivability in certain subsystems of the Logic of Proofs is $\Pi_{2}^{p}$-complete. Annals of Pure and Applied Logic, 145(3):223-239, March 2007.

18. Alexey Mkrtychev. Models for the logic of proofs. In Sergei Adian and Anil Nerode, editors, Logical Foundations of Computer Science, volume 1234 of Lecture Notes in Computer Science, pages 266-275. Springer Berlin Heidelberg, 1997.

19. Eric Pacuit. A note on some explicit modal logics. In Proceedings of the 5th Panhellenic Logic Symposium, Athens, Greece, 2005. University of Athens.

20. E. Spaan. Complexity of modal logics. PhD thesis, University of Amsterdam, 1993. 
21. Tatiana Yavorskaya (Sidon). Interacting explicit evidence systems. Theory Comput. Syst., 43(2):272-293, 2008. 